\title{
Article
}

\section{Synergetic theory of information}

\author{
Viktor Vyatkin \\ Independent researcher, 3 - 82 Papanina St., Ekaterinburg 620077, Russia \\ Correspondence: vbvvbv@yandex.ru
}

\begin{abstract}
A new approach is presented to defining the amount of information, in which information is understood as the data about a finite set as a whole, whereas the average length of an integrative code of elements serves as a measure of information. We obtain a formula for the syntropy of reflection, that is, the information that two intersecting finite sets reflect about each other. The specificities of reflection of discrete systems through the combination of their parts are considered, and it is shown that the additive syntropy and entropy of reflection are measures of structural order and chaos. Three information laws have been established: the law of conservation of the sum of chaos and order; information law of reflection; the law of conservation and transformation of information. An assessment of the structural organization and the level of development of discrete systems is presented. It is shown that various measures of information are structural characteristics of integrative codes of elements of discrete systems. The conclusion is made that, from the informational-genetic positions, the synergetic theory of information is primary in relation to the Hartley-Shannon information theory. In the appendix we consider the asymmetry of the mutual reflection of finite sets and the proportionality of arbitrary quantities.
\end{abstract}

Keywords: syntropy; entropy; chaos; order; amount of information; finite set; integrative code

\section{Introduction}

\begin{abstract}
"For the content, I found in the former metaphysics and logic only predominantly the external material. The philosophical depiction of the kingdom of thought, that is, in its necessary development, should therefore become a new enterprise, and, moreover, it should start all from the very beginning."
\end{abstract}

Hegel. Preface to the second edition of the book "Science of logic", 1831.

In the scientific and practical activity, we often deal with the discrete systems, the elements of which are described with the help of certain attributes. If the number of elements with a certain attribute is known, then we say that we have a finite set which is a part of the system. When two such sets $A$ and $B$, distinguished by the attributes $P_{A}$ and $P_{B}$ intersect each other $(A \cap B=K, K \neq \varnothing)$, we assert that these sets are interconnected and reflect certain information $I_{A B}$ about each other. In this case, we take into account the philosophical conclusion about the inseparable connection of information with reflection [1-3], and by the term reflection we means the reproduction of the features of one object through another object interacting with it.

The term information does not currently have an unambiguous scientific definition. Therefore, speaking of what should be taken as information in the context of reflection of sets through each other, we proceed from the usual understanding of information as knowledge or data about something. In this case, we consider sets $A$ and $B$ as integral entities and, accordingly, we say that information is the data about a finite set as a single whole. This definition of information extends not only to the mutual reflection of two sets, but also to the reflection of individual sets, both through themselves and through arbitrary collections of other sets. Therefore, in order to distinguish information $I_{A B}$ from other types of information, we consider it reasonable to give it a special 
name. This name, in our opinion, should take into account the fact that the interrelation of sets $A$ and $B$ is characterized by the fact that they interpenetrate each other and thereby form their joint image in the form of a connecting set $K$, the elements of which have the attributes of both sets. Considering the above, we will call information $I_{A B}$ the syntropy of reflection, bearing in mind that the Greek word syntropy can be translated as a mutual connection of images. (The first occasion that the word syntropy was used in this sense was in 1921 by the German pediatricians Pfaundler and Seht [4], who called syntropy "the mutual inclination, attraction" of two diseases.) That is, syntropy of reflection is the information that two intersecting finite sets reflects about each other as a single whole.

The quantitative assessment of the syntropy $I_{A B}$ of reflection is relevant in solving various problems in many subject areas. For example, in the problems of pattern recognition, syntropy $I_{A B}$ can be used as the informativeness of the attributes of the recognition objects, whereas in medical research it can be used to quantitatively characterize the interrelation of various diseases. At the same time, traditional approaches to determining the amount of information (Hartley's combinatorial approach [5], Shannon's probabilistic approach [6] and Kolmogorov's algorithmic approach [7]) do not allow performing an adequate assessment of syntropy $I_{A B}$. This is due to the fact that in the traditional approaches other types of information are considered. In the approaches of Hartley and Shannon, information is the removed uncertainty of choosing one of many possibilities, or, as Weaver said (the first popularizer of Shannon's theory), "information is a measure of one's freedom of choice when one selects a message" [8] (p. 9 ). In this case, the greater the uncertainty of the situation, the more information a concrete choice gives. Accordingly, in the case when the sets $A$ and $B$ correspond to each other in a one-to-one manner $(A=B)$ and there is no difference in the elements, then there is simply no choice among the elements, and the Hartley and Shannon approaches will show that $I_{A B}=0$. At the same time, it is obvious that, with a one-toone correspondence of the sets, the syntropy $I_{A B}$ should have the maximum possible value, that is, $A=B \Rightarrow I_{A B}=I_{A B}^{\max }$. In Kolmogorov's algorithmic approach, in turn, the length of the program for transforming one object into another is taken as information. Therefore, when $A=B$, the algorithmic approach will also show that $I_{A B}=0$. In other words, the attempts to define the quantity $I_{A B}$ using traditional approaches to quantifying information can lead to an apparent contradiction with common sense.

The indicated impossibility of assessing the syntropy $I_{A B}$ of reflection using the traditional approaches to determining the amount of information has initiated special information-theoretical studies, the result of which was a synergetic theory of information (STI). The subject of cognition of this theory is the information-quantitative aspects of the reflection of interconnected finite sets, as well as the specificities of the reflection of discrete systems through the totality of their parts. In this case, information is understood as the data about a finite set as a single whole, whereas the measure of information is the average length of an integrative code of elements. Regarding the name of the theory, we can say the following.

In STI, finite sets are considered as holistic formations. Accordingly, it is considered that the elements of the sets take part in the information processes of reflection as an integral collection, without individually selecting any of them as a separate event, test result, etc. Therefore, given that synergy means joint action, this word was used in the name of the theory. It should also be noted that in STI such aspects of the structure of discrete systems as chaos and order are considered, which brings this theory closer to the traditional synergetics of Haken [9]. Moreover, while in traditional synergetics, chaos and order are dynamic characteristics of the systems (the nature of the trajectory of the systems in the phase space is considered), in STI they characterize the own structure of the system in the "here and now" mode. That is, it can be said that STI and Haken's synergetic, with regard to the characteristics of discrete systems in terms of chaos and order, complement each other. Thus, we consider the name synergetic theory of information to be fully justified. 
The present paper outlines the basics of STI. It is noted that some fragments of the theory were previously published by the author in the form of a series of papers in Russian [10-14], whereas the presented material is a generalization and revision of these articles and contains new theoretical results.

The main content of the paper consists of seven sections 2-8 and is supplemented by Appendix A. Section 2 presents a new (synergetic) approach to defining the amount of information within which the formula for the syntropy $I_{A B}$ of reflection is obtained. Section 3 is devoted to the information analysis of the reflection of discrete systems through the totality of their parts, during which much attention is paid to such structural characteristics of systems as randomness and orderliness. In this case, two laws are established: the law of conservation of the sum of chaos and order and the information law of reflection of discrete systems. In Section 4, the law of the conservation and transformation into each other of various types of information (information attributive to control and information that exists independently of control) is substantiated and meaningfully interpreted. In Section 5 we consider the integrative codes of the elements of discrete systems and show the integrative-code interrelation between various measures of information. It is also concluded that, from an informational-genetic standpoint, the synergetic approach to defining the amount of information is primary relative to the combinatorial and probabilistic approaches. Sections 6 and 7 assess the structural organization and level of development of discrete systems. In this case, various types and variants of development are considered. Section 8 gives examples of the use of STI in areas such as medicine and linguistics. Appendix A, in turn, analyzes the information asymmetry of the mutual reflection of finite sets. Next, the asymmetry of reflection is associated with the commensuration of the sets, that is, with their correspondence to each other in terms of the number of elements.

\section{The synergetic approach to determining the amount of information}

\subsection{The problem of estimating the syntropy of reflection of finite sets}

The STI is based on the problem of quantitative estimation of the syntropy of reflection of finite sets. The formulation of this problem is as follows. - Suppose that within a certain discrete system $D=\{d\} \quad$ (Figure 1), two finite sets are distinguished by the attributes $P_{A}$ and $P_{B}$ : $A=\left\{a \mid P_{A}(a)\right\}=\left\{d \mid P_{A}(d)\right\}$ and $B=\left\{b \mid P_{B}(b)\right\}=\left\{d \mid P_{B}(d)\right\}$, such that $A \cap B=K$. The number of elements in the sets $A, B, K$ is, respectively, $|A|,|B|,|K|$. It is required to define the syntropy of reflection $I_{A B}$, that is, the amount of information that the sets $A$ and $B$ reflect about each other as a single whole.

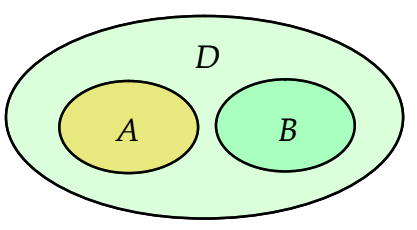

(a)

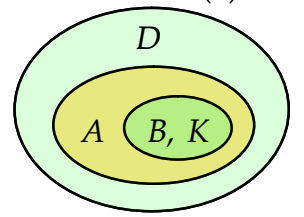

(c)

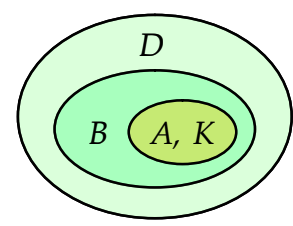

(d)

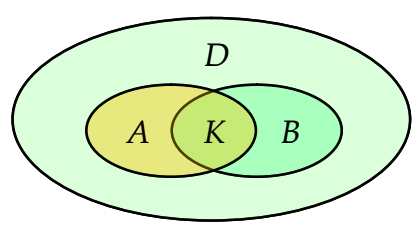

(b)

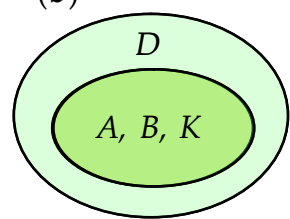

(e)

Figure 1. Interconnection of sets $A$ and $B$ in the system $D$ : (a) There is no relation $(K=\varnothing)$; (b) A partial relation $(K \neq \varnothing, K \neq A, K \neq B)$; (c) A partial relation $(K=B \subset A)$; (d) A partial relation ( $K=A \subset B)$; (e) A one-to-one relation $(A=B=K)$. 
Obviously, $I_{A B}=f(|A|,|B|,|K|)$. It is clear that if the values $|A|$ and $|B|$ are constant, the syntropy value $I_{A B}$ increases with the increase of $|K|$. It is also clear that if $K=\varnothing$ (Figure 1(a)), then $I_{A B}=0$; whereas $I_{A B}$ has its maximum value when $A=B=K$ (Figure $1(\mathrm{e})$ ). Accordingly, in the latter case, reflection of sets $A$ and $B$ through each other does not differ from their reflection through themselves, and the syntropy $I_{A B}$ is equal to the information that each set reflects about itself as an integral formation. That is,

$$
A=B=K \Rightarrow I_{A B}=I_{A}=I_{B}=I_{K}
$$

where $I_{A}, I_{B}, I_{K}$ is the information that the sets $A, B, K$ reflect about themselves.

It follows from the above that the value of syntropy $I_{A B}$ must satisfy the inequalities: $0 \leq I_{A B} \leq I_{A}$ and $0 \leq I_{A B} \leq I_{B}$. Therefore, we think that before solving the problem for the general case of intersection of sets $A$ and $B$ (Figures $1(b),(c),(d)$ ), it is necessary to define the amount of information that an arbitrary finite set $A$ reflects about itself.

It seems natural to define the values $I_{A B}$ and $I_{A}$ with the help of traditional information theory based on the work of Hartley [5] and Shannon [6]. At the same time, we have to admit that this cannot be done. The reason is that Hartley and Shannon treated information as the removed uncertainty of the choice of one of $N$ different possibilities. Moreover, Hartley considered the situation when all possibilities have the same probability $p_{i}=\frac{1}{N}, i=1,2, \ldots, N$ and proposed an information measure $H_{0}$ that, when using the binary logarithm, looks like:

$$
H_{0}=\log _{2} N \text {. }
$$

In turn, Shannon extended the views of Hartley to the general case, when $p_{i} \neq$ const, and, to measure information, he began to apply the entropy $H$ of the set of probabilities:

$$
H=-\sum_{i=1}^{N} p_{i} \log _{2} p_{i} .
$$

In this case, the contribution of the $i$-th opportunity to the value of $H$ is equal to its own information $I_{i}$, which is called "surprises" by Tribus [15]:

$$
I_{i}=-\log _{2} p_{i}
$$

If we try to use information measures (1) - (3) to determine $I_{A B}$ and $I_{A}$, then in the situation in Figure 1e we arrive at a contradiction with the common sense. In this situation, the sets $A$ and $B$ consist of the same elements, which do not differ in any way and there is no choice $(N=1, p=1)$. Accordingly, measures (1) - (3) will show that $I_{A}=0$ and $I_{A B}=0$, which cannot be. This suggests that, in the evaluation of syntropy $I_{A B}$ and information $I_{A}$, a new approach is required to the determination of the amount of information that should not be connected with the choice procedure. The development of this approach is presented below.

\subsection{Attributive information of a finite set}

Any arbitrary finite set $A$ has two attributive characteristics. This is the number of elements $|A|$ in the set and the attribute $P_{A}$ by which the set is distinguished. Therefore, assuming that $I_{A}=f(|A|)$, we will refer to information $I_{A}$ as attributive information. That is, attributive information $I_{A}$ is the information that the finite set $A$ reflects about itself. We consider the set $A$ as a single whole. This means that the elements of the set are not a mechanical collection of objects existing independently of each other, but an integral formation, uniting into which the elements acquire integrative properties that they do not have in their disjointed form. Taking this into 
account, in order to determine the value of attributive information $I_{A}$, we consider it reasonable to adopt two axioms: the axiom of monotonicity and the axiom of integrativity.

Axiom of monotonicity: Attributive information of a finite set is a monotonically increasing function of the total number of its elements, that is, for any two finite sets $A$ and $B$ with the number of elements $|A|$ and $|B|=|A|+1$ there holds the inequality

$$
I_{A}<I_{B} .
$$

Axiom of integrativity: An integrative code of the elements is a characteristic of a finite set $A$ as a single whole. In this case, the integrative code is an individual for each element sequence of the symbols of an alphabet, the number of which $L_{A}$ (the code length) is a function of the total number of elements $|A|$ in the set.

Let us now consider the process of increasing of the number $|A|$ of elements in the set $A$. We represent this process in the form of growth of an oriented tree, the set of suspended vertices of which is in one-to-one correspondence with the set $A$, while the maximum number of arcs emerging from one vertex is equal to the number of symbols (n) of the alphabet chosen for the compilation of integrative codes. In this case, each of the adjacent arcs is associated with its own symbol in alphabetical order, and the integrative code of an element is the sequence of symbols that are on the path of movement from the initial vertex of the tree to the suspended vertex corresponding to the given element. An example of such a code tree for $n=2$ when using an ordered pair of symbols $<0.1>$ as an alphabet is shown in Figure 2.

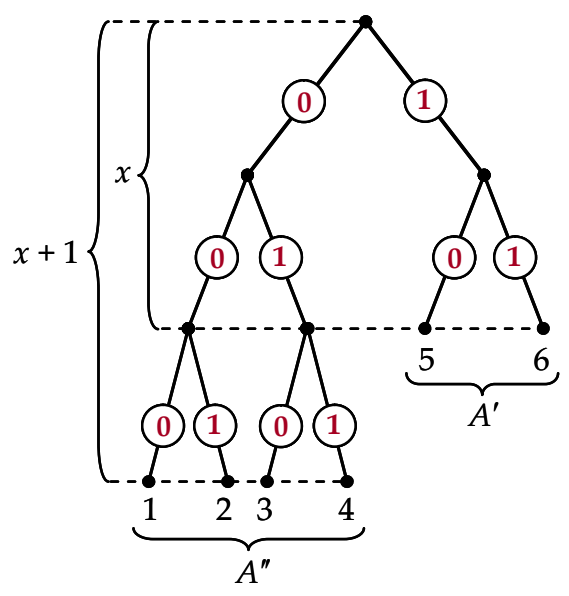

Figure 2. Code tree for $n=2$ and $|A|=6$.

The analysis of Figure 2 shows that, in the general case, the set $A$ is subdivided, with respect to the length of integrative codes $L_{A}$, into two subsets $A^{\prime} \subset A$ and $A^{\prime \prime} \subset A$ such that $L_{A^{\prime}}=x$ and $L_{A^{\prime \prime}}=x+1$, where $x=\left[\log _{n}|A|\right]$ is the integer part of $\log _{n}|A|$. That is, $L_{A}$ is not a single-valued function of $|A|$. Therefore, we will consider the average length of integrative codes

$$
\bar{L}_{A}=\frac{x\left|A^{\prime}\right|+(x+1)\left|A^{\prime \prime}\right|}{|A|}
$$

and start from an alphabet with the minimal number of symbols $(n=2)$.

It is seen from Figure 2 that for $n=2$ an increase of $|A|$ by one results in a decrease by one of the number of elements with the code length $x$ and an increase of the number of elements with the code length $x+1$ by two elements, that is:

$$
|A|+\left.1\right|_{n=2} \Rightarrow\left(\left|A^{\prime}\right|-1\right) \wedge\left(\left|A^{\prime \prime}\right|+2\right) .
$$


Let us determine the value of $\left|A^{\prime}\right|$ and $\left|A^{\prime \prime}\right|$; to this end, taking into account (6), we write the system of equations:

$$
\left\{\begin{array}{l}
\left|A^{\prime}\right|+\left|A^{\prime \prime}\right|=|A| \\
2\left|A^{\prime}\right|+\left|A^{\prime \prime}\right|=2^{x+1}
\end{array}\right.
$$

Solving this system, we get:

$$
\left\{\begin{array}{l}
\left|A^{\prime}\right|=2^{x+1}-|A| \\
\left|A^{\prime \prime}\right|=2\left(|A|-2^{x}\right)
\end{array}\right.
$$

Substituting the values of $\left|A^{\prime}\right|$ and $\left|A^{\prime \prime}\right|$ from (7) into (5), we arrive at the expression $\bar{L}_{A}$ for $n=2$ :

$$
\left.\bar{L}_{A}\right|_{n=2}=x+2-\frac{2^{x+1}}{|A|}
$$

The quantity $\bar{L}_{A}$ in equation (8) satisfies the adopted axioms and can serve as a measure of attributive information $I_{A}$.

Let us now consider the trees of codes for $n>2$. Figure 3 presents such trees when $n=3$ and $|A|=2,3,8,9$.

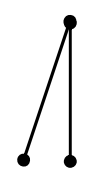

(a)

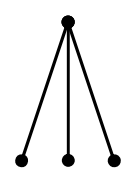

(b)

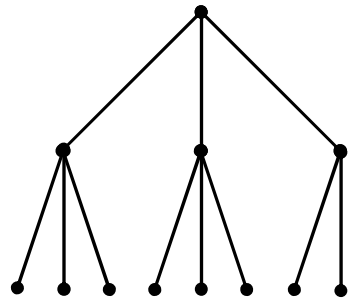

(c)

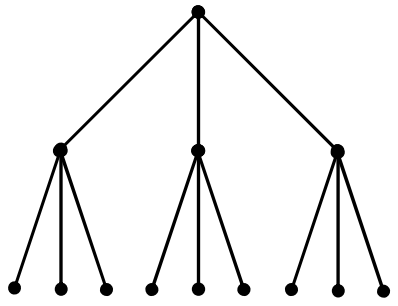

(d)

Figure 3. Code trees for $n=3:(\mathbf{a})|A|=2 ;(\mathbf{b})|A|=3 ;$ (c) $|A|=8 ;$ (d) $|A|=9$.

It is seen from Figure 3 that when the initial vertex of the tree (Figure 3(a), (b)) and the last of the suspended vertices (Figure 3(c), (d)) are filled with the outgoing arcs, the average code length $\bar{L}_{A}$ does not change, that is

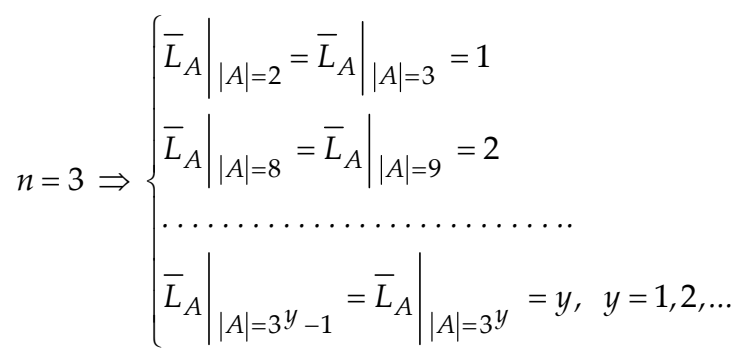

Increasing $n$, we arrive at the general expression of the cases when $\bar{L}_{A}$ retains its constant value for different values of $|A|$ :

$$
n>\left.2 \Rightarrow \bar{L}_{A}\right|_{|A|=n^{y}{ }_{-n+2}}=\left.\bar{L}_{A}\right|_{|A|=n^{y}{ }_{-n+3}}=\ldots=\left.\bar{L}_{A}\right|_{|A|=n} y=y .
$$

Expression (9) indicates that for $n>2$ the values of $\bar{L}_{A}$ contradict, in a number of cases, the monotonicity axiom (4). This allows us to draw an important conclusion: the average length of the 
integrative code of elements can act as a measure of the attribute information of the set only when the integrative codes are composed with the help of a binary alphabet.

Thus, we come to the conclusion that

$$
I_{A}=\left.\bar{L}_{A}\right|_{n=2}
$$

and all our further reasoning will apply only to the case $n=2$.

Analysis of equation (8) shows that if the equality $|A|=2^{x}$ is observed, then $\bar{L}_{A}=\log _{2}|A|$; whereas if the inequality $2^{x}<|A|<2^{x+1}$ holds, then $\log _{2}|A|<\bar{L}_{A}<1+\log _{2}|A|$. This suggests that, in the general case, $\bar{L}_{A}$ exceeds $\log _{2}|A|$ by a certain quantity, and this can be clearly seen in Figure 4.

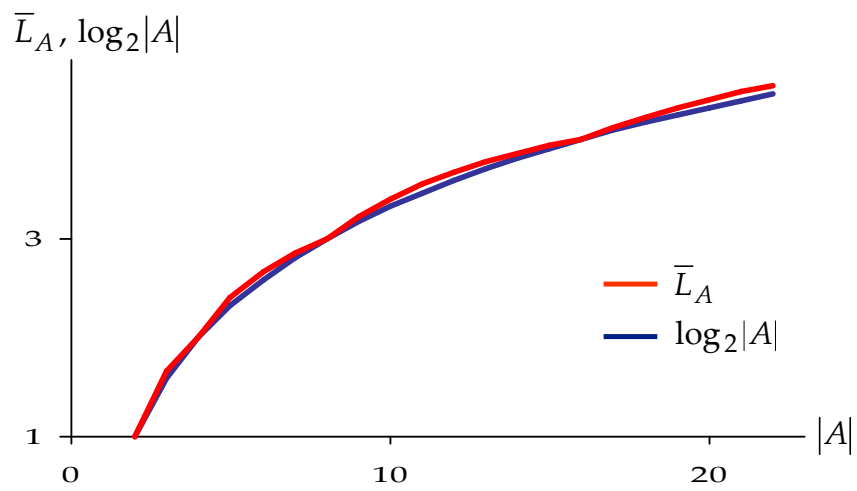

Figure 4. Dependence of $\bar{L}_{A}$ and $\log _{2}|A|$ on $|A|$.

We will determine the maximum value of this quantity as the exact upper bound $(\psi)$ of the difference $\bar{L}_{A}-\log _{2}|A|$ :

$$
\psi=\sup _{|A| \in[1, \infty)}\left(\bar{L}_{A}-\log _{2}|A|\right)
$$

Applying the necessary condition for the extremum of function and setting $|A| \in\left(2^{x}, 2^{x+1}\right)$, $x=$ const, we arrive, according to (8), at the equation

$$
\left(x+2-\frac{2^{x+1}}{|A|}-\log _{2}|A|\right)_{|A|}^{\prime}=0
$$

which after differentiation acquires the form

$$
\frac{2^{x+1}}{|A|^{2}}-\frac{1}{|A| \ln 2}=0
$$

and has a solution:

$$
|A|=2^{x+1} \ln 2 .
$$

Substituting the value of $\bar{L}_{A}$ from (8) and the value of $|A|$ from (12) into (11), we get: 


$$
\begin{aligned}
\psi & =x+2-\frac{2^{x+1}}{2^{x+1} \ln 2}-\log _{2}\left(2^{x+1} \ln 2\right) \\
& =1-\frac{1+\ln (\ln 2)}{\ln 2} \\
& =0.0860713 \ldots
\end{aligned}
$$

which is clearly illustrated by Figure 5 .

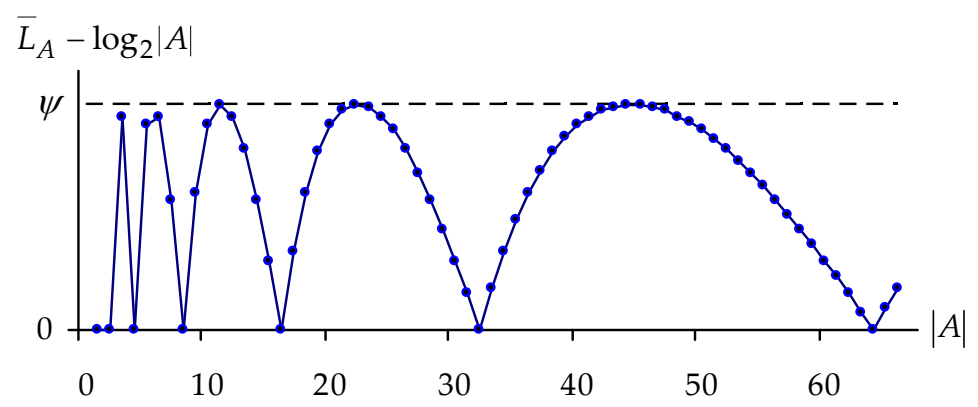

Figure 5. Dependence of the difference $\bar{L}_{A}-\log _{2}|A|$ on $|A|$.

The value of the obtained constant (13) allows making the approximation

$$
2^{x}<|A|<2^{x+1} \Rightarrow \bar{L}_{A} \approx \log _{2}|A|,
$$

considering which, we can say the following.

The function $f(|A|)=\log _{2}|A|,|A| \in[1, \infty)$ satisfies the axiom of monotonicity (4) and has a simpler form than $\bar{L}_{A}$ in equation (8). Therefore, in the sequel, for convenience of theoretical constructions and practical calculations, instead of (10) we will use its approximation:

$$
I_{A}=\log _{2}|A| \text {. }
$$

The obtained measure of attributive information (14) is mathematically similar to the information measure of Hartley (1), and at first glance it may seem that these measures do not differ from each other (the author has repeatedly encountered this opinion). Therefore, we will show by the example of letter sequences that $I_{A}$ and $H_{0}$ are similar to each other only in the external appearance of their equations (1) and (14). Such sequences and the corresponding values of $I_{A}$ and $H_{0}$ are given in Tables 1 and 2 .

Table 1. Information characteristics of letter sequences having the same length and a different variety of letters.

\begin{tabular}{cccccc}
\hline № & Letter sequence & $|\boldsymbol{A}|$ & $\boldsymbol{N}$ & $\boldsymbol{I}_{\boldsymbol{A}}$ & $\boldsymbol{H}_{0}$ \\
\hline 1 & $\mathrm{a}, \mathrm{b}, \mathrm{c}, \mathrm{d}, \mathrm{e}, \mathrm{f}, \mathrm{g}, \mathrm{h}$ & 8 & 8 & 3 & 3 \\
2 & $\mathrm{a}, \mathrm{a}, \mathrm{b}, \mathrm{b}, \mathrm{c}, \mathrm{c}, \mathrm{d}, \mathrm{d}$ & 8 & 4 & 3 & 2 \\
3 & $\mathrm{a}, \mathrm{a}, \mathrm{a}, \mathrm{a}, \mathrm{b}, \mathrm{b}, \mathrm{b}, \mathrm{b}$ & 8 & 2 & 3 & 1 \\
4 & $\mathrm{a}, \mathrm{a}, \mathrm{a}, \mathrm{a}, \mathrm{a}, \mathrm{a}, \mathrm{a}, \mathrm{a}$, & 8 & 1 & 3 & 0 \\
\hline
\end{tabular}

Table 2. Information characteristics of letter sequences having different lengths and the same variety of letters.

\begin{tabular}{cccccc}
\hline № & Letter sequence & $|\boldsymbol{A}|$ & $\boldsymbol{N}$ & $\boldsymbol{I}_{\boldsymbol{A}}$ & $\boldsymbol{H}_{0}$ \\
\hline 1 & $\mathrm{a}, \mathrm{b}$ & 2 & 2 & 1 & 1 \\
2 & $\mathrm{a}, \mathrm{a}, \mathrm{b}, \mathrm{b}$ & 4 & 2 & 2 & 1 \\
3 & $\mathrm{a}, \mathrm{a}, \mathrm{a}, \mathrm{a}, \mathrm{b}, \mathrm{b}, \mathrm{b}, \mathrm{b}$ & 8 & 2 & 3 & 1 \\
\hline
\end{tabular}


It can be seen from Table 1 that $I_{A}$ does not depend on the variety of letters $N$, forming the sequence; and it follows from Table 2 that $H_{0}$ does not depend on the length $|A|$ of the sequence. In other words, Tables 1 and 2 show that the attribute information of the set (14) and the Hartley information measure (1) are independent of each other and have different values in the same situation. Moreover, $H_{0} \leq I_{A}$, whereas equality $I_{A}=H_{0}$ is observed only in the particular case when $|A|=N$. All this suggests that, using equations (1) and (14), different types of information are measured.

\subsection{Units of information measurement}

The question naturally arises of the units of measurement of attributive information (14). Answering this question, first of all, we note that in the traditional measures of information $[5,6]$ the unit of measurement depends on the base of the logarithms used. The most widely used are binary logarithms, while the corresponding unit of information is bit, which is interpreted as the amount of information obtained by selecting one of two possibilities having the same probability. That is, according to the Shannon measure (2), 1 bit $=-\left(\frac{1}{2} \log _{2} \frac{1}{2}+\frac{1}{2} \log _{2} \frac{1}{2}\right)=\log _{2} 2$. The base of the logarithm in equation (14) can only be equal to two and, therefore, bit can serve as the unit of measurement of attributive information. In this case, one bit corresponds to the equality $I_{A}=1$ that is satisfied when $|A|=2$. At the same time, since attributive information is not related to the selection procedure, when measuring it, the bit must have a different meaning interpretation. Therefore, in order to distinguish between the results of measuring different types of information (associated with the choice and existing regardless of the choice), we will refer to the unit of measurement of attributive information as a reflection bit (abbreviated $r$-bit), which we define as follows: the reflection bit is the amount of information, which a set of two elements reflects about itself. That is, 1 r-bit $=\left.I_{A}\right|_{|A|=2}=\log _{2} 2$.

We note here that when two sets $A$ and $B$ are in one-to-one correspondence with one another $(A=B)$, then equality $I_{A B}=I_{A}=I_{B}$ holds. Therefore, we can say that $r$-bit is also a unit of measurement of the reflection syntropy $I_{A B}$.

\subsection{Syntropy of reflection}

We defined the value of the attributive information of a finite set as the average length of the integrative code of its elements (10), and we took the approximation (14) as the calculation formula. On this basis, the reflection syntropy $I_{A B}$ can be represented as the reproduction of the average length of the integrative code of the elements of each of the intersecting sets $A$ and $B$.

We now define the quantity $I_{A B}$ and assume that the sets $A, B, K$ form an information communication system in which the sets $A$ and $B$ are alternatively the source and receiver of information, whereas the connecting set $K=A \cap B$ acts as a transmission medium or communication channel. The process of the information transference through such a communication system corresponds to the process of reflection of the sets $A$ and $B$ through each other. Let us consider this process, assuming that each set takes part in it simultaneously with the totality of its elements. We first consider the reflection of the set $A$ through the set $B$.

The integrative code of any element $a \in A$ is a certain message about a finite set $A$ as a holistic entity. The average length of such messages equals $I_{A}$, and their total number is equal to $|A|$. Accordingly, the amount of information $V_{A}$ sent by the reflected set $A$ as a source of information to the connecting set $K$ as a communication channel equals:

$$
V_{A}=I_{A}|A| \text {. }
$$


The carrying capacity of the set $K$, in turn, is bounded by the product $I_{K}|K|$; and, accordingly, the amount of information $V_{A \rightarrow K}$ that the set $K$ can take from the set $A$ is equal to this product. That is:

$$
V_{A \rightarrow K}=I_{K}|K|
$$

Since $|K| \leq|A|$, then $V_{A \rightarrow K} \leq V_{A}$ and, in the general case, the messages from the set $A$ enter the set $K$ in a truncated form. That is, the information $I_{A}$ when entering the set $K$ is reduced to the value

$$
I_{A \rightarrow K}=\frac{V_{A \rightarrow K}}{|A|}=I_{K} \frac{|K|}{|A|} .
$$

Only $|K|$ messages can come to the reflecting set $B$ from the connecting set $K$; each message contains the information $I_{A \rightarrow K}$. This means that the following amount of information comes from the set $A$ to the set $B$ as a receiver of information:

$$
V_{A \rightarrow B}=I_{A \rightarrow K}|K|=I_{K} \frac{|K|^{2}}{|A|}
$$

which is distributed over all elements $b \in B$. That is, the information $I_{A}$ is reproduced by the set $B$ in the form of information $I_{A \rightarrow B}$, which has the following form:

$$
I_{A \rightarrow B}=\frac{V_{A \rightarrow B}}{|B|}=I_{K} \frac{|K|^{2}}{|A| \cdot|B|} .
$$

If we now consider the inverse process of reflecting the set $B$ through the set $A$, then we obtain exactly the same result (15). It follows that

$$
I_{A \rightarrow B}=I_{B \rightarrow A}=I_{A B}
$$

and the reflection syntropy $I_{A B}$, according to (14) and (15), equals:

$$
I_{A B}=\frac{|K|^{2}}{|A| \cdot|B|} \log _{2}|K| \text {. }
$$

Equation (16) refers to the most general case of intersection of sets $A$ and $B$, when $K \neq A$ and $K \neq B$ (Figure 1(b)). In more particular cases, when $K=B \subset A$ (Figure $1(\mathrm{c})$ ) and $K=A \subset B$ (Figure $1(\mathrm{~d}))$, the syntropy $I_{A B}$ can be represented in the following form:

$$
\begin{aligned}
& K=B \subset A \Rightarrow I_{A B}=\frac{|B|}{|A|} \log _{2}|B|, \\
& K=A \subset B \Rightarrow I_{A B}=\frac{|A|}{|B|} \log _{2}|A| .
\end{aligned}
$$

When obtaining equation (16), we have verified that the sets $A$ and $B$ reflect the same amount of information about each other. At the same time, the number of elements in the composition of these sets is generally different, which causes asymmetry $(A s)$ of the completeness of their reflection through each other. This asymmetry can be represented as the absolute value of the difference between the relative values of the reflection syntropy $I_{A B}$ for each of the sets. That is, denoting the relative syntropy of the reflection of the set $A$ by the symbol $I_{A \rightarrow B}^{*}$, and for the set $B$, by the symbol $I_{B \rightarrow A}^{*}$, we get:

$$
I_{A \rightarrow B}^{*}=\frac{I_{A B}}{I_{A}}
$$




$$
\begin{gathered}
I_{B \rightarrow A}^{*}=\frac{I_{A B}}{I_{B}}, \\
A s=\left|I_{A \rightarrow B}^{*}-I_{B \rightarrow A}^{*}\right|=I_{A B}\left|\frac{1}{I_{A}}-\frac{1}{I_{B}}\right| .
\end{gathered}
$$

The reflection asymmetry (21) has a number of features connected to the relation between the sets $A$ and $B$ with respect to the number of elements that are considered in Appendix $A$ of the present paper.

Thus, having obtained equations (16)-(18), we thereby solved the formulated problem of estimating the reflection syntropy $I_{A B}$, and actually developed a new approach to determining the amount of information which is not connected with a procedure of choice. At the same time, in our reasoning, the sets $A, B, K$ participated in information processes together with the totality of their elements. Therefore, we will call this new approach a synergetic approach.

\subsection{Comparative analysis of the relations between finite sets}

Concluding the presentation of the basics of a synergetic approach to defining the amount of information, we dwell on the aspects of its use in solving problems related to the analysis of the relations between finite sets. In the first approximation, such problems can be divided into two types. In the first type of problems, the studied collection of sets must be ordered according to the strength of the relation with some particular set. (A typical example of this type of problems is the assessment of the informativeness of the attributes of reference objects in the pattern recognition algorithms.) The second type of problems is characterized by the fact that a comparative analysis of the relationship of various different pairs of sets is carried out. (Solving such problems is of great importance, for example, in clinical medicine in the complex analysis of the relations between diseases.)

From the standpoint of the synergetic approach, the first type of problems can be solved using only the syntropy of reflection (16). For example, if the sets $A, B, C, D$ are given and it is required to determine which of the sets $B, C, D$ is related to the largest degree with the set $A$, then the solution of the problem will be the set whose reflection through the set $A$ has the maximum value. At the same time, if all the sets $A, B, C, D$ intersect with one another and the second type of the problem is posed - to determine which two sets are most strongly interconnected, then, using only formula (16), we can come to a wrong solution. That is, for example, for the sets $A$ and $B$ when $|A|=|B|=|K|=16$, we get $I_{A B}=4$, whereas for the sets $C$ and $D$ such that, $|C|=34,|D|=36$, $|K|=32$, we have $I_{C D}=4.18$. Here concluding that a stronger relation is observed between sets $C$ and $D$ than between sets $A$ and $B$, we make a mistake, because sets $A$ and $B$ correspond to each other in one-to-one manner and therefore are interconnected to the maximum extent, while the sets $C$ and $D$ have no such correspondence.

From this, it follows that, in general, in order to objectively compare the relations between various pairs of sets, it is necessary to take into account the relative value of the reflection syntropy of each set (19) and (20). The measure of interrelation in this case is the average relative syntropy $I_{A B}^{*}$ of both sets:

$$
I_{A B}^{*}=\frac{I_{A \rightarrow B}^{*}+I_{B \rightarrow A}^{*}}{2}=\frac{I_{A B}}{2}\left(\frac{1}{I_{A}}+\frac{1}{I_{B}}\right), \quad 0 \leq I_{A B}^{*} \leq 1
$$

Using (22) to solve the problem of the second type in the above example, we get $I_{A B}^{*}=1$, $I_{C D}^{*}=0.82$; that is, sets $A$ and $B$ are more strongly interconnected with each other than sets $C$ and D. 


\section{Information aspects of the reflection of discrete systems}

\subsection{Introductory remarks}

In the previous section, we carried out an information analysis of the reflection of two intersecting finite sets $A$ and $B$ through each other. Let us now consider the reflection of set $A$ through the collection of sets $B_{1}, B_{2}, \ldots, B_{N}$ distinguished in the system $D$ by the values of the characteristic $P_{B}=P_{B_{1}}, P_{B_{2}}, \ldots, P_{B_{N}}$ and assume that $\bigcup_{i=1}^{N} B_{i} \geq A, \bigcap_{i=1}^{N} B_{i}=\varnothing, \quad A \cap B_{i}=K_{i} \neq \varnothing$ (Figure 6). In this case, for convenience, we will consider set $A$ as a discrete system, and consider set $K_{i}$ as a part of this system.

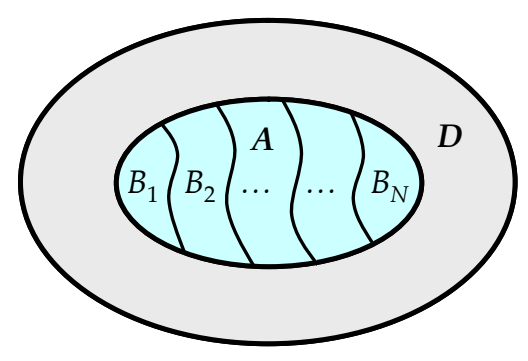

(a)

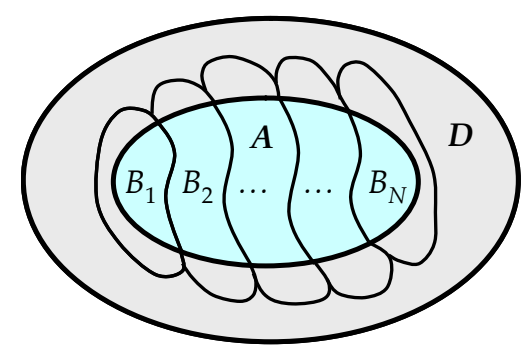

(b)

Figure 6. Reflection of system $A$ by a collection of sets $B_{1}, B_{2}, \ldots, B_{N}$ : (a) system $A$ is informationally closed; (b) system $A$ is informationally open.

We also note that system $A$ with respect to the environment (elements of the complementary set $C=D \backslash A=\{d \in D, d \notin A\})$ can be both closed and open in informational terms. That is, system

$A$ is informationally closed if $\bigcup_{i=1}^{N} B_{i}=A$ (Figure 6(a)) and informationally open if $\bigcup_{i=1}^{N} B_{i}>A$ (Figure 6(b)).

Taking into account the comments made, we proceed to the information analysis of the reflection of system $A$ through the collection of sets $B_{1}, B_{2}, \ldots, B_{N}$ and begin with the case when system $A$ is informationally closed.

\subsection{Reflection of informationally closed systems}

\subsubsection{Additive syntropy and entropy of reflection}

The first thing we encounter when analyzing the informational aspects of the reflection of system $A$ is its attributive information $I_{A}$, which the system reflects about itself as a holistic entity. This information is reflected by the system through its $N$ parts $K_{1}, K_{2}, \ldots, K_{N}$, which, in the case of informational closeness of the system, coincide with the sets $B_{1}, B_{2}, \ldots, B_{N}$, that is, $K_{i}=B_{i}$. At the same time, each part $K_{i}$ reflects a certain amount of information about the system, which is equal to syntropy $I_{A B_{i}}$, which according to (17) has the form:

$$
I_{A B_{i}}=\frac{\left|K_{i}\right|}{|A|} \log _{2}\left|K_{i}\right|
$$

Accordingly, the total amount of information about system $A$, which is actually reproduced through $N$ of its parts, is the additive syntropy $I_{\Sigma}$ of reflection, equal to the sum of partial syntropies (23): 


$$
I_{\Sigma}=\sum_{i=1}^{N} \frac{\left|K_{i}\right|}{|A|} \log _{2}\left|K_{i}\right|
$$

Let us compare the information being reflected $\left(I_{A}\right)$ and the information that has been reflected $\left(I_{\Sigma}\right)$. To this end, we multiply the right-hand sides of equations (14) and (24) by $|A|$ and divide by $\log _{2}|A|$. As a result of this operation, for $N>1$ we get the inequality

$$
|A|>\sum_{i=1}^{N}\left|K_{i}\right| \frac{\log _{2}\left|K_{i}\right|}{\log _{2}|A|}
$$

from which it follows that

$$
I_{A}>I_{\Sigma}
$$

Inequality (25) says that not all information about system $A$, as a single whole, is reflected through the totality of its parts, and there is always some part of information $I_{A}$ that remains unreflected. Denote this non-reflected information by the symbol $S$ and define its value as the difference between the information being reflected and the information that has been reflected:

$$
S=I_{A}-I_{\Sigma}
$$

Let us represent (26) in expanded form and carry out simple transformations:

$$
\begin{aligned}
S & =\log _{2}|A|-\sum_{i=1}^{N} \frac{\left|K_{i}\right|}{|A|} \log _{2}\left|K_{i}\right| \\
& =\log _{2}|A|-\sum_{i=1}^{N} \frac{\left|K_{i}\right|}{|A|} \log _{2} \frac{\left|K_{i}\right|}{|A|}-\log _{2}|A| \sum_{i=1}^{N} \frac{\left|K_{i}\right|}{|A|} \\
& =-\sum_{i=1}^{N} \frac{\left|K_{i}\right|}{|A|} \log _{2} \frac{\left|K_{i}\right|}{|A|} .
\end{aligned}
$$

If we now multiply the right-hand side of equation (27) by the total number $|A|$ of elements of the system and use natural logarithms, then we get a mathematical copy of statistical weight of a thermodynamic system, which is a measure of its entropy [16]. This circumstance gives us a reason to call the unreflected information $S$ the entropy of reflection. That is, the entropy of reflection is the information about a discrete system that is not reflected (not reproduced) through a collection of its parts. In other words, entropy $S$ characterizes inadequacy, uncertainty of reflection of a discrete system through its parts, while the additive syntropy $I_{\Sigma}$ is a measure of adequacy, definitiveness of this reflection.

Until now, in our reasoning, we have never used such a concept as probability. We just did not need to. At the same time, the quotient $\frac{\left|K_{i}\right|}{|A|}$ can be interpreted from the standpoint of the theory of probability as the probability $p_{i}$ of encountering the elements with the characteristic $P_{B_{i}}$ among all the elements of system $A$. With this interpretation of the indicated quotient, the entropy of reflection $S$ is represented as follows:

$$
S=-\sum_{i=1}^{N} p_{i} \log _{2} p_{i}
$$

Comparison of (2) and (28) shows that the reflection entropy $S$ and the Shannon entropy $H$ do not differ mathematically from each other, that is,

$$
S=H .
$$

Equality (29) indicates that STI and Shannon's theory of information, though dealing with different types of information, are at the same time mathematically interrelated. The nature of this 
relationship, as will be shown in Section 5.2, has an integrative code character. Moreover, we note the following.

In Shannon's theory, certain requirements are a priori imposed on the entropy $H$ of the set of probabilities [6], and the entire theory, in fact, begins with the derivation of formula (2). In STI, in turn, no preliminary conditions are set for the entropy of reflection $S$; it is obtained simply as the difference between the information being reflected $I_{A}$ and the information that has been reflected $I_{\Sigma}$. That is, in the chronological sequence of obtaining information-synergetic functions $I_{A} \rightarrow I_{A B} \rightarrow I_{\Sigma} \rightarrow S$, the entropy of reflection $S$ is the closing member. This fact suggests that the synergetic information with which STI is dealing is genetically primary with respect to the probabilistic information, as measured in Shannon's theory (we will come to the same conclusion, but from other standpoints, in section 5.2). This is fully consistent with Kolmogorov's conclusion that "information theory should precede probability theory, but not rely on it" [17] (p. 35). That is, STI can be viewed as verification of this conclusion of a recognized authority in the field of information theory.

\subsubsection{Chaos and order of discrete systems}

The structure of any discrete system with a finite set of elements can be characterized with the help of such concepts as order and disorder or chaos. At the same time, these concepts are not unambiguously defined in modern science. Therefore, starting a conversation about informational analysis of chaos and order in the structure of discrete systems, we first take a decision concerning the interpretation that will be used in the further presentation of our material.

So, we say that there is a complete order in the structure of the system in terms of the values of a certain attribute $P_{B}=P_{B_{1}}, P_{B_{2}}, \ldots, P_{B_{N}}$, if all elements have the same value of this attribute. That is, when all the elements in the composition of the system do not differ from each other in terms of this attribute, we say that the structure of the system is maximally ordered. In contrast, complete chaos in the structure of the system occurs when each element of the system has its own individual value of the attribute $P_{B}=P_{B_{1}}, P_{B_{2}}, \ldots, P_{B_{N}}$. That is, in this case, all the elements are different from each other, and we say that the structure of the system is maximally chaotic.

What has been said is formalized by the fact that, for complete order, $N=1$, and for complete chaos, $N=|A|$. In other cases, when $1<N<|A|$, the system is in an intermediate state between complete chaos and complete order, and we say that the structure of the system is characterized by both chaos and orderliness. In this case, if the system changes its state in such a way that $N \rightarrow|A|$, then order develops into chaos, whereas if $N \rightarrow 1$, then the chaos is transformed into order. Taking into account the comments made, we now turn to the information analysis of the structural features of discrete systems in terms of chaos and order.

An analysis of (24) and (27) shows that the value of the additive syntropy $I_{\Sigma}$ and the entropy of reflection $S$ depends on the number of parts of the system and their mutual relations according to the number of elements. At the same time, the more chaotic the structure of the system is, that is, the more parts are identified in its composition and the less the parts differ from each other in terms of the number of elements, the larger is $S$ and the smaller is $I_{\Sigma}$. Conversely, the more order is in the structure of the system, that is, the smaller is the number of its parts and the more the parts differ from each other in terms of the number of elements, the larger is $I_{\Sigma}$ and the smaller is $S$. This indicates that $I_{\Sigma}$ and $S$ depend on the degree of orderliness and randomness of the structures of discrete systems and can be considered as measures of order and chaos. Let us show this using a concrete example.

Figure 7 shows various states of a discrete system consisting of 16 elements, which are characterized by the "direction of motion" characteristic. According to the values of this characteristic, the system successively takes 5 states, which correspond to its division into 1, 2, 4, 8, 16 parts which have the same number of elements. 


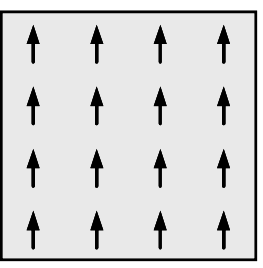

(a)

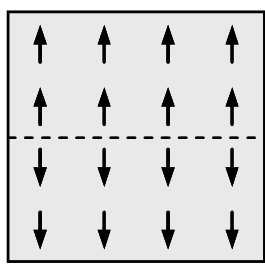

(b)

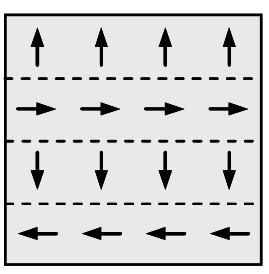

(c)

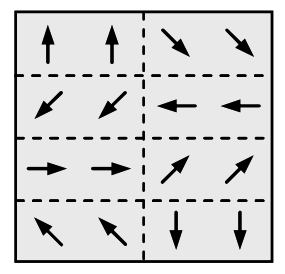

(d)

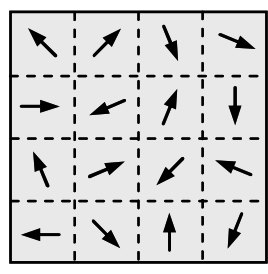

(e)

Figure 7. Division of a system of 16 elements into equal parts according to the "direction of motion" attribute: (a) $N=1$; (b) $N=2$; (c) $N=4 ;$ (d) $N=8 ;$ (e) $N=16$.

In the state of the system in Figure 7(a), all elements move in the same direction and an ideal order is observed in the structure of the system. In Figure 7(e) we have the polar opposite, that is, each element of the system has an individual direction of movement, and the structure of the system is maximally chaotic. The states of the system in Figures $7(b),(c),(d)$ are intermediate to those in Figures 7(a),(e) and are characterized by the fact that both chaos and orderliness are observed in the structure of the system. Calculating by (24) and (27) for each state of the system the values of additive syntropy and entropy of reflection, we successively obtain: $I_{\Sigma}=4,3,2,1,0$; $S=0,1,2,3,4$. That is, an increase in chaos and a decrease in order in the structure of the system in Figure 7 correspond to a decrease in the values of $I_{\Sigma}$ and an increase in the values of $S$.

Additive syntropy $I_{\Sigma}$, as the reflected information, and the entropy of reflection $S$, as nonreflected information, are integral parts of the reflected information $I_{A}$, that is:

$$
I_{A}=I_{\Sigma}+S .
$$

At the same time, as was shown, the values of $I_{\Sigma}$ and $S$ characterize the structure of a discrete system from the standpoints of order and chaos. Therefore, equation (30) can be also interpreted as the following expression:

$$
\operatorname{Order}\left(I_{\Sigma}\right)+\operatorname{Chaos}(S)=\text { const }\left(I_{A}\right) \text {. }
$$

That is, it can be argued that equation (30) and its interpretation (31) can be viewed as the law of conservation of the sum of chaos and order, which is valid for any structural transformation of the system without changing the total number of its elements. In other words, no matter what we do with the system without changing the total number of its elements, no matter into how many parts the system is divided by the values of any characteristic and whatever relations in terms of the number of elements are between the parts, the sum of chaos and order in the system structure will always remain constant. For example, for all the states of the system in Figure 7, there holds the equality: $I_{\Sigma}+S=4$.

Let us now consider the specific features of the relationship between additive syntropy $I_{\Sigma}$ and entropy of reflection $S$ with a constant number of elements of the system and a different number of its parts, correlated with each other in the number of elements in an arbitrary way. First, in accordance with equations (14), (24), (27), (30), we define the maximum and minimum values of $I_{\Sigma}$ and $S$ for a fixed $N$.

When $N=|A|$ all parts of the system consist of only one element and, according to (24), we have $I_{\Sigma}=0$. Thus, it follows from (30) that, in this case, $S=I_{A}$. In other words, for $N=|A|$ syntropy $I_{\Sigma}$ assumes the minimal value, and entropy $S$, the maximum of all possible values for any $N$. Since in this case all parts of the system are equal with one another in terms of the number of elements $\left(\left|K_{i}\right|=1\right)$, then in all other cases, when $N<|A|$ and $\left|K_{i}\right|=\frac{|A|}{N}$, we should expect that syntropy $I_{\Sigma}$ is minimal, and entropy $S$, maximal. That is:

$$
S^{\max }=-N \frac{1}{N} \log _{2} \frac{1}{N}=\log _{2} N .
$$




$$
I_{\Sigma}^{\min }=\log _{2}|A|-\log _{2} N=\log _{2} \frac{|A|}{N} .
$$

In turn, additive syntropy will assume its maximum value when the number of elements in one part is equal to $|A|-N+1$, and each of the other $N-1$ parts includes only one element, that is:

$$
I_{\Sigma}^{\max }=\frac{|A|-N+1}{|A|} \log _{2}(|A|-N+1) .
$$

Accordingly, the minimum entropy of reflection is equal to:

$$
S^{\min }=\log _{2}|A|-\frac{|A|-N+1}{|A|} \log _{2}(|A|-N+1) .
$$

We construct the graphs of dependence of $I_{\Sigma}^{\min }, I_{\Sigma}^{\max }, S^{\min }, S^{\max }$ on the number $N$ of parts of the system with $|A|=$ const and analyze the resulting diagram, which we will call the information field of reflection of discrete systems (Figure 8).

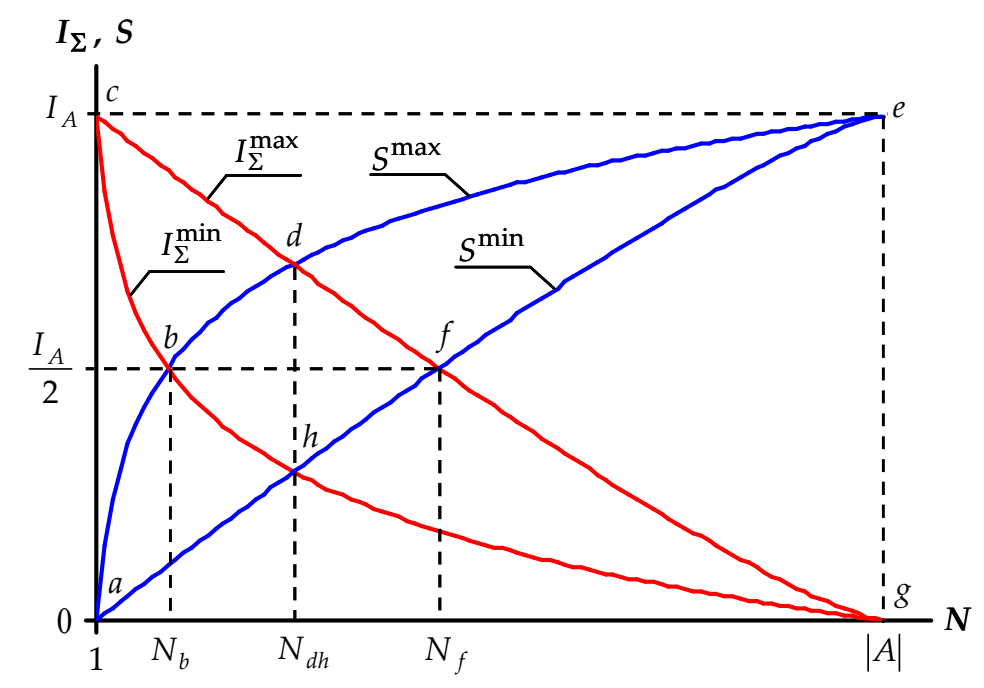

Figure 8. Information field of reflection of discrete systems $(|A|=100)$.

The graphs constructed in the figure initially form two contours - the entropic one abdefha and the syntropic one $c d f g h b c$, which localize the ranges of all possible values of $I_{\Sigma}$ and $S$. The intersection of these contours at the points $b$ and $f$, where equalities $I_{\Sigma}^{\min }=S^{\max }$ and $I_{\Sigma}^{\max }=S^{\min }$ are observed, allows, using the projection of these points on the horizontal axis, to identify three intervals of values of $N$ : the left $\left(1 \leq N<N_{b}\right)$, the central $\left(N_{b} \leq N \leq N_{f}\right)$, and the right $\left(N_{f}<N \leq|A|\right)$ ones. Let us consider the features of relationships between $I_{\Sigma}$ and $S$ in these intervals and first let us determine the values of $N_{b}$ and $N_{f}$.

At point $b$, according to (32) and (33), we have the equation $\log _{2} N_{b}=\log _{2} \frac{|A|}{N_{b}}$, the solution of which yields $N_{b}=\sqrt{|A|}$ (hereinafter, the values of $N$ are rounded to the nearest larger integer). A similar equation for the point $f$ formed by the right-hand sides of (34) and (35) has no analytical solution. That is, the value of $N_{f}$ can only be determined numerically, which is as follows. For the members of the sequence $N=1,2, \ldots,|A|$ are determined by $I_{\Sigma}^{\max }$ and $S^{\min }$, and as the value of $N_{f}$ we take the value of $N$, after which $S^{\min }>I_{\Sigma}^{\max }$. The values $N_{f}$ calculated in this way for 
$|A|=50,100, \ldots, 500$ are shown in Table 3, where the corresponding values of $N_{b}$ are also given. Using these values, the regression equation $\hat{N}_{f}=0.44|A|$ is obtained, and the values of $\hat{N}_{f}$ calculated from it for comparison with the values of $N_{f}$ are also presented in the indicated table. In our further reasoning, we will use this regression equation for a general characteristic of the quantity $N_{f}$.

Table 3. The values of $N_{b}, N_{d h}, \hat{N}_{d h}, N_{f}, \hat{N}_{f}$ for $|A|=50,100, \ldots, 500$.

\begin{tabular}{cccccc}
\hline$|A|$ & $N_{b}$ & $N_{d h}$ & $\hat{N}_{d h}$ & $N_{f}$ & $\hat{N}_{f}$ \\
\hline 50 & 8 & 14 & 15 & 21 & 22 \\
100 & 10 & 25 & 25 & 44 & 44 \\
150 & 13 & 36 & 36 & 66 & 66 \\
200 & 15 & 47 & 46 & 88 & 88 \\
250 & 16 & 58 & 57 & 111 & 110 \\
300 & 18 & 68 & 67 & 133 & 132 \\
350 & 19 & 78 & 78 & 156 & 154 \\
400 & 20 & 88 & 88 & 179 & 176 \\
450 & 22 & 98 & 99 & 201 & 198 \\
500 & 23 & 108 & 109 & 224 & 220 \\
\hline
\end{tabular}

Thus, for $|A|=$ const, we have three intervals of values of $N$ :

- the left one: $1 \leq N<\sqrt{|A|}$;

- the central one: $\sqrt{|A|} \leq N \leq 0.44|A|$;

- the right one: $0.44|A|<N \leq|A|$.

The systems that fall into the left interval according to the value of $N$ are characterized by the fact that for any relations between their parts in terms of the number of elements, the inequality $I_{\Sigma}>S$ is preserved. That is, in the left interval of the values of $N$, the structural orderliness of the system is always greater than its randomness. In the right interval, the opposite situation is observed, when inequality $S>I_{\Sigma}$ holds for any structural transformation of the system, which corresponds to the predominance of chaos over order in the structure of the system. In the central interval, there is an intersection of the regions of the possible values of $I_{\Sigma}$ and $S$, as a result of which there can be various relations between them $\left(I_{\Sigma}>S, I_{\Sigma}<S, I_{\Sigma}=S\right)$, and there is a tendency towards structural harmony of chaos and order.

In other words, in the left interval of the values of $N$, we observe an irreversible predominance of order over chaos in the structure of the system, and in the right interval, on the contrary, irreversible predominance of chaos over order. In the central interval, in turn, the predominance of both order over chaos and chaos over order is reversible. Any discrete system with a finite set of elements, divided by the values of any characteristic into $N$ parts, inevitably falls into one of the specified intervals, which allows all systems to be classified into three types according to the specific features of these intervals:

- $\quad$ ordered (left interval);

- harmonious (central interval);

- $\quad$ chaotic (right interval).

For example, the system in Figure 7, consisting of 16 elements, in its various states will be classified as follows: (a) ordered $(N=1<\sqrt{16})$; (b) ordered $(N=2<\sqrt{16})$; (c) harmonious $(N=4=\sqrt{16})$; (d) chaotic $(N=8>0.44 \cdot 16)$; (e) chaotic $(N=16>0.44 \cdot 16)$.

The division of the values of $N$ into left, center, and right intervals is not comprehensive. In addition to this division, the central interval is additionally subdivided into left $\left(N_{b} \leq N \leq N_{d h}\right)$ 
and right $\left(N_{d h}<N \leq N_{f}\right)$ parts according to the value $N_{d h}$, which corresponds to the combined projection of the points $d$ and $h$. We note the features of these parts, and first we determine the value $N_{d h}$.

At the point $d$, the equality $I_{\Sigma}^{\max }=S^{\max }$ is observed, and at the point $h$, the equality $I_{\Sigma}^{\min }=S^{\min }$. It follows that the value $N_{d h}$ must satisfy the equation:

$$
I_{\Sigma}^{\max } \cdot S^{\min }-I_{\Sigma}^{\min } \cdot S^{\max }=0
$$

Equation (36) has no analytical solution, as a result, the values $N_{d h}$ can only be obtained numerically (by analogy with the above determination of $N_{f}$ ). Such numerical solutions for $|A|=50,100, \ldots, 500$ lead to a regression equation $\hat{N}_{d h}=0.21|A|+4$, and the corresponding values of $N_{d h}$ and $\hat{N}_{d h}$ are given in Table 3 . In what follows, we will use this regression equation for a general characteristic of the values $N_{d h}$.

Thus, the left and right parts of the central interval have the form:

- $\quad$ the left part: $\sqrt{|A|} \leq N \leq 0.21|A|+4$;

- the right part: $0.21|\mathrm{~A}|+4<N \leq 0.44|\mathrm{~A}|$.

The interrelationships of syntropy $I_{\Sigma}$ and entropy $S$ in the left and right parts of the central interval are characterized by the fact that in the left part, $S^{\min }<I_{\Sigma}^{\min }<S^{\max }<I_{\Sigma}^{\max }$, and in the right part, $I_{\Sigma}^{\min }<S^{\min }<I_{\Sigma}^{\max }<S^{\max }$. That is, in the structure of systems from the left part there is a tendency for dominance of order over chaos, which weakens with increasing of $N$, and in the structure of systems from the right part, the opposite tendency for the prevalence of chaos over order is manifested, which increases together with the increase of $N$. These features of interrelations between $I_{\Sigma}$ and $S$ in the left and right sides of the central interval, as well as the equality $I_{\Sigma}=S$, observed in each of these parts along the $b f$ line, provide a ground for further dividing harmonious systems into three kinds:

- $\quad$ ordered harmonious (the left part of the central interval);

- balanced harmonious (the entire central interval with $I_{\Sigma}=S$ );

- $\quad$ chaotic harmonious (the right part of the central interval).

Thus, all discrete systems with a finite set of elements are divided into three types according to the proportion of chaos $(S)$ and order $\left(I_{\Sigma}\right)$ in their structure; these types include five sorts of systems.

\subsection{Reflection of informationally open systems}

When the system is informationally open (Figure $6(\mathrm{~b})$ ), it interacts with the environment. As a result of this interaction, part of the information $I_{A}$ being reflected passes into the environment and is distributed among the elements of the complementary sets $B_{1} \backslash K_{1}, \ldots, B_{N} \backslash K_{N}$, which, together with the parts $K_{1}, \ldots, K_{N}$ of the system, form reflecting objects in the form of the sets $B_{1}, \ldots, B_{N}$. In this case, the reflection of the system as a whole becomes more uncertain and diffused. Accordingly, in relation to the informationally closed state, the additive syntropy of the reflection of the system decreases, while the entropy of reflection increases.

Obviously, in accordance with the general definition of syntropy (16), the additive syntropy $I_{\Sigma}^{*}$ of the informationally-open state of the system has the form

$$
I_{\Sigma}^{*}=\sum_{i=1}^{N} \frac{\left|K_{i}\right|^{2}}{|A| \cdot\left|B_{i}\right|} \log _{2}\left|K_{i}\right|,
$$


and, in the case $\bigcup_{i=1}^{N} B_{i}=A$, is reduced to the additive syntropy $I_{\Sigma}$ of the informationally closed state. The entropy $S$ of reflection, in turn, is an invariant quantity with respect to any relations of the system with the environment (this follows from the fact that the ratio $\frac{\left|K_{i}\right|}{|A|}$ in equation (27) does not depend on the degree of openness of the system). The latter circumstance suggests that, with the informational opening of the system, a new component $\Delta S$ appears in the entropy of its reflection, which characterizes the relationship of the system with the environment. In accordance with this, in order to meaningfully differentiate between $S$ and $\Delta S$, we will use the terms internal $(S)$ and external $(\Delta S)$ entropy of reflection.

We define the value of external entropy $\Delta S$ based on the balance between the information $\left(I_{A}\right)$ being reflected, the information $\left(I_{\Sigma}^{*}\right)$ that has been reflected and the non-reflected information $(S+\Delta S)$. Since the information $\left(I_{A}\right)$ being reflected and the internal entropy $S$ do not change during the informational opening of the system, the external entropy $\Delta S$ is defined as the difference between additive syntropies of the informationally closed $\left(I_{\Sigma}\right)$ and informationally open $\left(I_{\Sigma}^{*}\right)$ states:

$$
\begin{aligned}
\Delta S & =I_{\Sigma}-I_{\Sigma}^{*} \\
& =\sum_{i=1}^{N} \frac{\left|K_{i}\right|}{|A|} \log _{2}\left|K_{i}\right|-\sum_{i=1}^{N} \frac{\left|K_{i}\right|^{2}}{|A| \cdot\left|B_{i}\right|} \log _{2}\left|K_{i}\right| \\
& =\sum_{i=1}^{N} \frac{\left|B_{i}\right|-\left|K_{i}\right|}{\left|K_{i}\right|} \cdot \frac{\left|K_{i}\right|^{2}}{|A| \cdot\left|B_{i}\right|} \cdot \log _{2}\left|K_{i}\right| .
\end{aligned}
$$

The first factor under the sign of the last sum in (38) is the ratio of the number of elements of the complementary set $B_{i} \backslash K_{i}$ to the number of elements of the connecting set $K_{i}=A \cap B_{i}$. That is, this factor characterizes the relationship of the system with the environment from the side of the set $B_{i}$, that is why, we will call it the coefficient of informational openness of the system $\left(\mu_{B_{i}}\right)$ with respect to the characteristic $P_{B_{i}}$ :

$$
\mu_{B_{i}}=\frac{\left|B_{i}\right|}{\left|K_{i}\right|}-1,0 \leq \mu_{B_{i}}<\infty
$$

The combination of the second and third factors, in this case, represents the syntropy $I_{A B_{i}}^{*}$ of the reflection of an open system through the set $B_{i}$. Therefore, taking into account (39), the final expression of the external entropy $\Delta S$ of reflection has the form:

$$
\Delta S=\sum_{i=1}^{N} \mu_{B_{i}} I_{A B_{i}}^{*} .
$$

Let us return to the consideration of the information field of reflection of discrete systems in Figure 8. Obviously, the rectangular contour acega limits the total area of the information field, which includes all possible values of additive syntropy and entropy of reflection for both informationally open and closed systems. In Section 3.2.2 it was shown that if the system is informationally closed, then the areas of possible values of additive syntropy and reflection entropy are limited by the corresponding contours $c d f g h b c$ and abdefha. When the system informationally opens, its reflection becomes more uncertain and the areas of possible values of both additive syntropy and entropy increase. Moreover, the new values of additive syntropy $\left(I_{\Sigma}^{*}\right)$ are located below the graph of $I_{\Sigma}^{\min }$ and, with an unlimited increase in the information openness of the system $\left(\forall \mu_{B_{i}} \rightarrow \infty\right)$, they asymptotically approach the line $a g$, while the values of the total entropy of 
reflection $(S+\Delta S)$ are above the graph of $S^{\max }$ and have as their the asymptote the line ce. Thus, the areas of possible values of the reflected and non-reflected information are limited, in the general case, by the following contours: the additive reflection syntropy, by acdfga, the reflection entropy, by acefha. Completing the description of the distribution of the information $I_{A}$ being reflected over the information field acega of the reflection, we also note that within it there is a vast area efge, into which the values of both additive syntropy and reflection entropy never fall.

\subsection{Information law of reflection}

Let us work out the information balance of reflection of discrete systems in the general case:

$$
I_{A}=I_{\Sigma}^{*}+S+\Delta S
$$

Equation (41) is a stable, single-valued relation between the information being reflected, the information that has been reflected and the non-reflected information, which makes it possible to give it the status of a law. Let us call this law the informational law of reflection and formulate it as follows: when reflecting a discrete system $A$ through a collection of finite sets $B_{1}, B_{2}, \ldots, B_{N}$, such that $\bigcup_{i=1}^{N} B_{i} \geq A, \bigcap_{i=1}^{N} B_{i}=\varnothing$, there takes place a division of the reflected information into reflected and nonreflected parts, the first of which is equal to additive syntropy, and the second is the sum of the internal and external entropy of reflection. In the case, when the system is in the informationally closed state $\left(\forall \mu_{B_{i}}=0\right.$ ), equation (41) reduces to equation (30) and the non-reflected part of the information is equal only to the internal entropy $S$ of reflection.

\section{The law of conservation and transformation of information}

The information law of reflection (41) is similar in its form to the known physical laws of conservation (of energy, electric charge, etc.). Already in the 50s and 60s of the last century, mathematicians and philosophers spoke about the need to establish such laws in the theory of information $[3,18]$. At that, special attention was paid to the fact that conservation laws in information theory should take into account the mutual transitions of different types of information into each other. Such information transitions can be seen in analyzing equation (41) if, in accordance with equation (29), we consider the internal entropy of reflection $S$ as Shannon's probabilistic information $H$, which is associated with a choice among many possibilities, whereas the additive syntropy $I_{\Sigma}^{*}$ and the outer entropy $\Delta S$ are considered as some kinds of synergetic information that exists independently of this choice. In this case, equation (41) takes the form

$$
I_{A}=I_{\Sigma}^{*}+H+\Delta S
$$

and is interpreted as the law of conservation and transformation of information, which is formulated as follows: the total amount of synergetic and probabilistic information, which characterize the structure of a discrete system and its relationships with the environment, is constant provided the number of elements of the system is fixed. Let us reveal the content of this law separately for informationally closed and informational open systems, for which we present them in graphical form, as shown in Figure 9. 


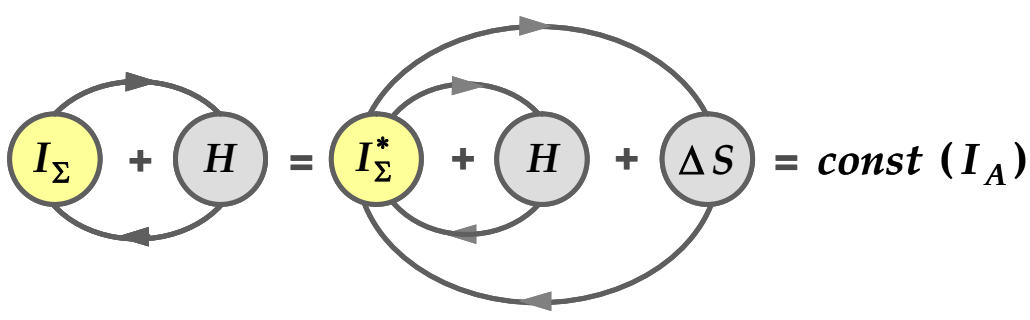

(a)

(b)

Figure 9. Graphic expression of the law of conservation and transformation of information: (a) informationally closed system; (b) informationally open system.

Informationally closed systems. When the system is informationally closed, the law of conservation and transformation of information (42) appears before us in its particular form

$$
I_{A}=I_{\Sigma}+H,
$$

and the relations between different types of information, as well as their mutual transitions into each other, are determined by the direction in which the structural transformations of the system occur. So, if the number of parts of the system decreases, and even more so if at the same time some part of the system begins to dominate in terms of the number of elements, then the amount of synergetic information $I_{\Sigma}$ increases and the amount of probabilistic information $H$ decreases.

In the limit, when the division of the system into parts ceases $(N=1, p=1)$, its probabilistic information is completely transformed into synergetic information and, as such, ceases to exist in the system, that is, $I_{\Sigma}=I_{A}, H=0$. In this case, there is no variety of elements and possibilities of choice. In the case of inverse structural transformations, when the number of parts of the system increases, the opposite information process takes place and the synergetic information turns into the probabilistic information. This process exhausts itself and ends when the number of parts of the system becomes equal to the number of its elements $\left(N=|A|, p_{i}=1 /|A|\right)$ and, correspondingly, $I_{\Sigma}=0, H=I_{A}$. The variety of elements and possibilities of choices are maximal in this situation.

Informationally open systems. When the system informationally opens, its interaction with the environment begins; as a result of that, some of the information reflected by the system goes into the environment in the form of the external entropy $\Delta S$ of reflection. If the system further begins to close again $\left(\forall \mu_{B_{i}} \rightarrow 0\right)$, then this information is returned to the system in the form of the corresponding increase in the values of $I_{\Sigma}^{*}$. Since $\Delta S<I_{\Sigma}$, then, irrespective of the degree of increase in the informational openness of the system $\left(\forall \mu_{B_{i}} \rightarrow \infty\right)$, only a limited amount of information can go to the external environment. From this it follows that the system cannot give to the external environment, and also get back from the external environment an amount of information that exceeds the additive syntropy of reflection of its informationally closed state.

Also, as in the case of informationally closed systems, we consider two opposite structural states of the system. In the first case, when the system is maximally ordered $(N=1)$, only synergetic information is manifested in it and before the system is opened we have $I_{\Sigma}^{*}=I_{\Sigma}=I_{A}$, $\Delta S=0$. After informational opening of the system and its unbounded growth $\left(\forall \mu_{B_{i}} \rightarrow \infty\right)$, practically all the information reflected by the system is disseminated over the environment and, accordingly, $I_{\Sigma}^{*} \rightarrow 0, \Delta S \rightarrow I_{A}$. In the second case, with the maximum randomness of the structure $(N=|A|)$, all information of the system is represented only by a probabilistic form, which is invariant with respect to relations of the system with the external environment. Accordingly, both before the opening of the system and after its opening, equality $H=I_{A}$ is preserved, indicating 
that, if the structure of the system is maximally chaotic, then for any degree of openness of the system, there is no information exchange with the environment, and $I_{\Sigma}^{*}=0, \Delta S=0$. That is, reaching the highest possible level of structural chaos, the system becomes invisible to the external environment.

\section{Information measures in the light of integrative codes}

In Section 2 it was shown that the measure of attributive information of a finite set is the average length of the integrative code of its elements. Now we show what other information measures represent in terms of integrative codes both in the STI and in the Hartley-Shannon information theory.

\subsection{Integrative codes of the elements of discrete systems}

Let us consider two situations concerning finite sets $B_{1}, B_{2}, \ldots, B_{N}$ that are identified in a universal set $U$ by means of an attribute $P_{B}=P_{B_{1}}, P_{B_{2}}, \ldots, P_{B_{N}}$. In the first situation, the sets are isolated from each other and exist by themselves, whereas in the second situation, the sets are combined into a system $A=\bigcup_{i=1}^{N} B_{i}$ and each of them is an independent part of this system.

When the set $B_{i}$ is isolated from other sets, its elements have the average length $\bar{L}_{B_{i}}$ of an integrative code, and when the sets form a system $A$, then in the composition of this system the integrative codes of elements of any set have the average length $\bar{L}_{A}$. Since $\left|B_{i}\right| \leq|A|$, then $\bar{L}_{B_{i}} \leq \bar{L}_{A}$. This means that when combining sets into a system, the average length of the integrative code of their elements increases by the value of the difference $\bar{L}_{A}-\bar{L}_{B_{i}}$. It follows that the integrative code of any element of system $A$ is generally divided into two parts. In this case, the first part is formed due to the set of elements having the same value of the attribute $P_{B}$, whereas the second part is a superstructure over the first and is due to the combination of the collection of sets $B_{1}, B_{2}, \ldots, B_{N}$ into a system. Since any set $B_{i}$ in the composition of the system $A$ is a homogeneous group of elements with respect to $P_{B_{i}}$, we give the indicated parts of integrative codes the following names: the first part is the group part of the code, the second part is the system part of the code. In addition, the length of these parts will be denoted by the following symbols: $L^{g}$ is the length of the group part, $L^{s}$ is the length of the system part.

Thus, the length $L$ of the integrative code of any element of a discrete system, in the general case, can be represented as follows:

$$
L=L^{g}+L^{S} .
$$

In order to have a visual representation of the division of integrative codes into the group and system parts, we refer to Figure 10, where the code trees of a discrete system $A$ with the number of elements $|A|=8$ are shown. (Such a graphic illustration of the division of codes into parts is possible only when the logarithms $\log _{2}|A|$ and $\log _{2}\left|B_{i}\right|$ have integer values.) The figure shows three situations that reflect two polar (a), (c) and one common (b) cases of division of the system into $N$ parts. 


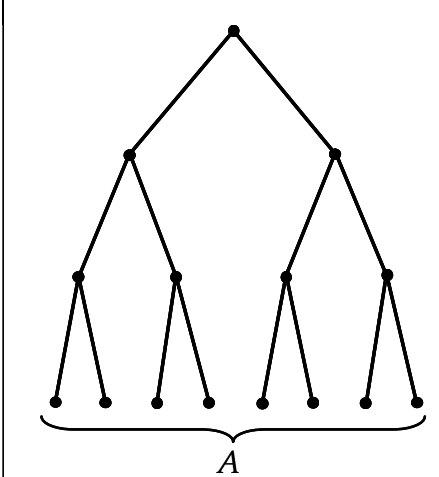

(a)

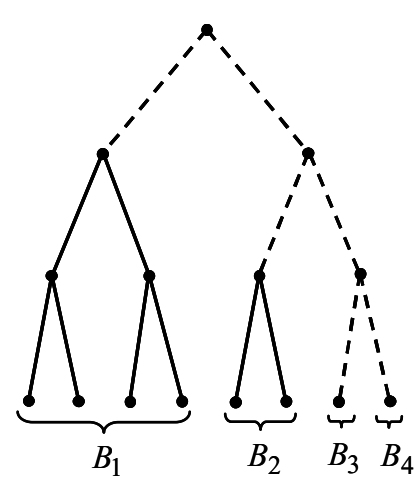

(b)

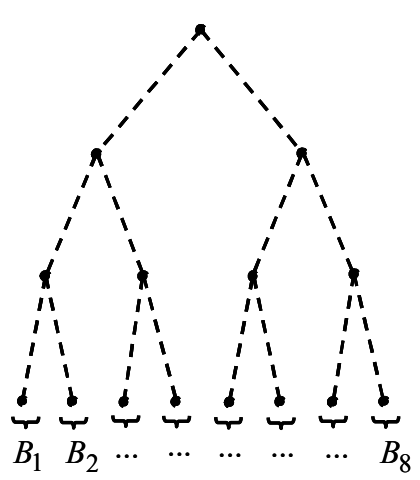

(c)

the group part of the code; $\stackrel{-\bullet}{\bullet}$ the system part of the code

Figure 10. The trees of integrative codes of a discrete system $A$ with the number of elements $|A|=8$ : (a) there is no division of the system into parts $(N=1) ;(\mathbf{b})$ the general case of dividing the system into parts $(1<N<|A|)$; (c) the number of parts of the system is equal to the number of its elements $(N=|A|)$.

In Figure 10(a), there is no division of the system into parts $(N=1)$, that is, all elements of the system have the same value of the attribute $P_{B}$, and accordingly, all codes are represented only by the group part $L^{g}$. The opposite situation is shown in Figure 10(c), where the number of parts of the system is equal to the number of its elements $(N=|A|)$, that is, in this case, each element of the system has an individual value of the attribute $P_{B}$, and its integrative code consists only of the system part $L^{S}$. Figure $10(\mathrm{~b})$ shows the most general case of dividing a system into parts $(1<N<|A|)$, when the number of elements in any part satisfies the inequality $1 \leq\left|B_{i}\right| \leq|A|-N+1$. In this case, if $\left|B_{i}\right|=1$ (parts $B_{3}$ and $B_{4}$ ), then the code is represented only by the system part $L^{S}$, and if $\left|B_{i}\right|>1$ (parts $B_{1}$ and $B_{2}$ ), then in the structure of the code, both the group part $L^{g}$ and the system part $L^{S}$ are distinguished.

Let us now consider the quantitative aspects of integrative codes of elements in the composition of discrete systems, and let us begin with determining the size of the group part and the total length of the code. From (10) and (14) it follows that the average length $\bar{L}_{A}$ of the integrative code of the elements of system $A$ and the average value $\bar{L}_{B_{i}}^{g}$ of the group part of the code of the elements of any part $B_{i}$ are equal to:

$$
\begin{gathered}
\bar{L}_{A}=\log _{2}|A|, \\
L_{B_{i}}^{g}=\log _{2}\left|B_{i}\right| .
\end{gathered}
$$

The weighted average value $\bar{L}_{A}^{g}$ of the length of the group part of the code for all parts of the system, in turn, has the form: 


$$
\begin{aligned}
\bar{L}_{A}^{g} & =\frac{\sum_{i=1}^{N}\left|B_{i}\right| \log _{2}\left|B_{i}\right|}{\sum_{i=1}^{N}\left|B_{i}\right|} \\
& =\sum_{i=1}^{N} \frac{\left|B_{i}\right|}{|A|} \log _{2}\left|B_{i}\right| .
\end{aligned}
$$

From (44)-(46) it follows that the average length $\bar{L}_{B_{i}}^{s}$ of the system part of the code of the elements of any part $B_{i}$ is equal to:

$$
\bar{L}_{B_{i}}^{s}=\bar{L}_{A}-\bar{L}_{B_{i}}^{g}=-\log _{2} \frac{\left|B_{i}\right|}{|A|} .
$$

Accordingly, the weighted average value $\bar{L}_{A}^{s}$ of the system part of the integrative code over all elements of system $A$ has the form:

$$
\begin{aligned}
\bar{L}_{A}^{s} & =\frac{-\sum_{i=1}^{N}\left|B_{i}\right| \log _{2} \frac{\left|B_{i}\right|}{|A|}}{\sum_{i=1}^{N}\left|B_{i}\right|} \\
& =-\sum_{i=1}^{N} \frac{\left|B_{i}\right|}{|A|} \log _{2} \frac{\left|B_{i}\right|}{|A|} .
\end{aligned}
$$

In the case when all parts of the system are equal, that is, when $\left|B_{i}\right|=|A| / N$, we have:

$$
\left.\overline{L_{A}^{s}}\right|_{\left|B_{i}\right|=\text { const }}=\log _{2} N \text {. }
$$

Thus, we have established that the integrative codes of the elements of discrete systems are divided, in the general case, into the group and system parts, and also have determined the quantitative characteristics of these parts.

\subsection{Integrative-code interpretation of the information measures}

An analysis of the obtained characteristics of integrative codes, as they are compared with the information measures of combinatorial, probabilistic and synergetic approaches, allows us to give these measures the following interpretation.

Combinatorial and probabilistic approaches. The most widely known measure of information, the entropy of the Shannon probability set (2), is the weighted average of the system part of the integrative code of the elements of the discrete system (49):

$$
H=\bar{L}_{A}^{S} \text {. }
$$

When all parts of the system are equal, the combinatorial Hartley measure (1) is also expressed in terms of the weighted average of the system part of the code:

$$
H_{0}=\left.\bar{L}_{A}^{S}\right|_{\left|B_{i}\right|=\text { const }} \text {. }
$$

The self-information (3), appearing in the probabilistic approach, in turn, represents the average value of the system part of the code (48) of those elements of the system that have the same value of the attribute $P_{B}$, that is: 


$$
I_{i}=\bar{L}_{B_{i}} .
$$

Synergetic approach. The measure of attribute information (14), initially introduced in this approach, by definition (10), is the average length of the integrative code of all elements of both a single finite set and a discrete system as a whole. In this case, when $N=1$, the integrative codes of the system are represented only by the group part and, accordingly,

$$
I_{A}=\bar{L}_{A}=\left.\bar{L}_{A}^{g}\right|_{N=1} .
$$

The partial syntropy of the reflection of the system through any part of (17) is the corresponding term of the last sum in (47) and, taking into account (46), it is equal to

$$
I_{A B_{i}}=\frac{\left|B_{i}\right|}{|A|} \bar{L}_{B_{i}}^{g} .
$$

The additive syntropy of reflection (24), in turn, is equal to the weighted average value of the group part of the code of all elements in the system (47):

$$
I_{\Sigma}=\bar{L}_{A}^{g} .
$$

The entropy of reflection of the system through the collection of its parts (26), as well as the Shannon entropy (2), is equal to the weighted average value of the system part of the integrative code of the elements of the system (49), that is:

$$
S=\bar{L}_{A}^{S} .
$$

A summary of what has been said is Table 4, which presents the information measures of the combinatorial, probabilistic, and synergetic approaches, as well as their integrative-code interpretation.

Table 4. Information measures and their integrative-code interpretation

\begin{tabular}{|c|l|l|}
\hline \multirow{2}{*}{$\begin{array}{c}\text { Approach to the } \\
\text { definition of the } \\
\text { amount of } \\
\text { information }\end{array}$} & \multicolumn{2}{|c|}{ Information measure } \\
\cline { 2 - 3 } & \multicolumn{1}{|c|}{ Initial form } & \multicolumn{1}{c|}{$\begin{array}{c}\text { Integrative-code } \\
\text { interpretation }\end{array}$} \\
\hline Combinatorial & $H_{0}=\log _{2} N$ & $H_{0}=\left.\bar{L}_{A}^{s}\right|_{\left|B_{i}\right|=\text { const }}$ \\
\hline \multirow{4}{*}{ Probabilistic } & $H=-\sum_{i=1}^{N} p_{i} \log _{2} p_{i}$ & $H=\bar{L}_{A}^{s}$ \\
\cline { 2 - 3 } & $I_{i}=-\log _{2} p_{i}$ & $I_{i}=\bar{L}_{B_{i}}^{s}$ \\
\hline \multirow{4}{*}{ Synergetic } & $I_{A}=\log _{2}|A|$ & $I_{A}=\bar{L}_{A}=\bar{L}_{A}^{g} \mid$ \\
\cline { 2 - 3 } & $I_{A B_{i}}=\frac{\left|B_{i}\right|}{|A|} \log _{2}\left|B_{i}\right|$ & $I_{A B_{i}}=\frac{\left|B_{i}\right|}{|A|} \bar{L}_{B_{i}}^{g}$ \\
\cline { 2 - 3 } & $I_{\Sigma}=\sum_{i=1}^{N} \frac{\left|B_{i}\right|}{|A|} \log _{2}\left|B_{i}\right|$ & $I_{\Sigma}=\bar{L}_{A}^{g}$ \\
\cline { 2 - 3 } & $S=-\sum_{i=1}^{N} \frac{\left|B_{i}\right|}{|A|} \log _{2} \frac{\left|B_{i}\right|}{|A|}$ & $S=\bar{L}_{A}^{s}$ \\
\hline
\end{tabular}

The above Table clearly shows that the information measures of the combinatorial and probabilistic approaches are functionally related only to the system part of the integrative code of 
the elements, while the synergetic approach includes both parts of the code together with its total length. In this case, the system part of the code is formed only when the individual sets are combined into a system, or when a homogeneous system begins to divide into parts. That is, prior to this union or division, the integrative codes are represented only by the group part, and there is only the attribute information of individual sets or a homogeneous system. It follows that the information considered in the synergetic approach is genetically primary with respect to the information measured in the combinatorial and probabilistic approaches. This is a confirmation of the conclusion made in Section 3.2.1 of the primacy of synergetic information, which was based on the sequence of obtaining information-synergetic functions $I_{A}, I_{A B}, I_{\Sigma}, S$.

The general conclusion that can be made as a result of analysis of Table 4 is that the information measures of combinatorial, probabilistic and synergetic approaches are in their essence quantitative characteristics of the structural features of integrative codes of the elements of discrete systems. Figuratively speaking, one can say that these informational measures are masks behind which the characteristics of integrative codes are hidden. Moreover, the entropy $S$ of reflection and the Shannon entropy $H$ are two different masks of the weighted average value of the system part of the code. All this suggests that the synergetic information theory and information theory in the Hartley-Shannon version in their totality form a unified theory of the quantitative aspect of the phenomenon of information.

\section{Structural organization of discrete systems}

It is obvious that the structural organization of a discrete system depends on the randomness and orderliness of its structure. Moreover, the more order in the structure of the system, the more the system is organized, and vice versa, the more disorder, the less the level of organization is. Accordingly, to quantify the structural organization of systems, one or another function can be used, the arguments of which are measures of chaos and order. As such a function of the structural organization, we can use the ratio of additive syntropy $I_{\Sigma}$ to the entropy of reflection $S$, which will be called $R$-function ( $R$ is the first letter of the word reflection):

$$
R=\frac{I_{\Sigma}}{S}=\frac{\sum_{i=1}^{N}\left|K_{i}\right| \log _{2}\left|K_{i}\right|}{|A| \log _{2}|A|-\sum_{i=1}^{N}\left|K_{i}\right| \log _{2}\left|K_{i}\right|} .
$$

The greater the values of the $R$-function, the higher the structural organization of the system is. In this case, the values of $\mathrm{R}$ indicate what and to what extent predominates in the structure of the system: chaos or order. For example, if $R>1$, then order prevails in the structure of the system, otherwise, when $R<1$, chaos. When $R=1$, chaos and order balance each other, and the structural organization of the system are equilibrium. Accordingly, in the left interval of the values of $N$, systems always have $R>1$, in the right interval, $R<1$, whereas in the central interval, the structural organization of the systems may be diverse, that is, $R>1, R<1$ or $R=1$. In this case, when all elements of the system have one and the same characteristic, that is, when $N=1$ and $S=0$, it is assumed that $R=\infty$. For example, for the states of the system in Figure 7, whose syntropy $I_{\Sigma}$ and entropy $S$ were determined above, we have the following values: (a) $R=\infty$; (b) $R=3$; (c) $R=1$; (d) $R=0,33$; (e) $R=0$.

It should be noted that the $R$-function, represented as

$$
R=\frac{I_{A}-S}{S},
$$

in its appearance is similar to the informational Shannon's redundancy $H^{*}$ [6], which Foerster [19] proposed to use as a measure of the organization of systems: 


$$
H^{*}=\frac{H^{\max }-H}{H^{\max }},
$$

where $H$ is the actual entropy of the system (2), $H^{\max }$ is the maximum entropy that a system can have for a given variety of its $N$ elements. In this case, Foerster connected the organization of the system only with the order and believed that the order should be considered relative to the maximum disorder. That is, if $H=H^{\max }$, then $H^{*}=0$ and there is no order or organization in the system, whereas if $H=0$, then $H^{*}=1$, and the system is maximally ordered and organized.

Since $H=S$ and $H^{\max }=H_{0}$, while the mathematical expressions for $H_{0}$ and $I_{A}$ are outwardly similar to each other, it may seem that it does not matter much which equation (59) or (60) to use when evaluating the organization of systems. But it is not so, which is easily seen by turning again to the states of the system in Figure 7. Here, when analyzing the states (a) and (e), using equations (59) and (60), we, in fact, arrive at the same conclusions that in state (a) the system is maximally organized ( $N=1, R=\infty, H^{*}=1$ ), whereas in state (e) it is completely disorganized ( $\left.N=|A|, R=0, H^{*}=0\right)$. At the same time, the analysis of the states (b), (c), (d), where $1<N<|A|$, leads to different conclusions. That is, from the standpoint of (60), the system in all these states has the same value $H^{*}=0$ and is disorganized as in state (e), which is not true. Equation (59), in turn, gives different values of $R$-function (which are given above), according to which, in the process of transition of the system from state (b) to state (d), its structural organization decreases. In other words, from the standpoint of (60), systems with entropy $H=H^{\text {max }}$ do not differ from each other in their organization, but from the positions of (59) they differ, whereas the magnitude of the differences depends on the values of $|A|$ and N. In this regard, it is pertinent to note that Foerster, while suggesting evaluation of the order and organization of systems using Shannon's redundancy, warned that this may not always be useful.

The differences in the assessment of the organization of systems with the help of $H^{*}$ and $R$ are explained by the fact that redundancy of Shannon's information $H^{*}$ characterizes the system only as a source of symbolic messages and shows that when $p_{i}=$ const there is no redundancy and the receiver of information (the cognizing subject) is all the time in a state of maximum uncertainty regarding the next symbol of the message. In this case, the message structure does not play any role and is not taken into account. In contrast, when we evaluate the organization of the system using the $R$-function, we, on the contrary, are only interested in the structure of messages and do not care about any properties of the source of these messages.

\section{Structural development of discrete systems}

\subsection{Types of structural development}

Apart from structural organization, discrete systems are also characterized by the level of their development, which is related to the ratio of chaos and order in the structure of the system and depends on the total number of system elements, the number of its parts and the distribution of the latter in terms of the number of elements. In this respect, three types of structural development can be distinguished: intensive, extensive and complex. The intensive type of development is characterized by the fact that the number of elements in the composition of the system remains constant all the time, while the number of system parts in the process of development increases, that is, $|A|=$ const , $N \rightarrow|A|$. In contrast, under the extensive type of development, the number of parts of the system does not change, while the total number of elements increases all the time, that is, $|N|=$ const, $|A| \rightarrow \infty$. The complex type of development, in turn, combines elements of the first two types and is manifested in the fact that, as the total number of system elements increases, the number of its parts also increases, that is, $|A| \rightarrow \infty, N \rightarrow|A|$. In order to better comprehend the indicated types of systems development, we present some specific examples. 
An example of the intensive development of systems is the structural transformation of an isolated system of ideal gases during its transition from an ordered state with zero entropy to a state of thermodynamic equilibrium with maximum entropy. A general idea of the extensive development of systems, in turn, can be obtained using the example of writing a sizeable text, if we consider the text as a discrete system, whose elements are letters, while the parts are sets of identical letters. In this case, after using all the letters of the alphabet in the text, the further development of the text as a system will be expressed in an unlimited increase in the total number of elements with a constant number of parts. In this case, until the moment of using up the entire alphabet, the development of the text will be complex, that is, an increase in the total number of letters will be accompanied by an increase in the number of parts of the text. Additionally, as a physical example of a complex type of system development, we point out to the evolution of electronic systems of atoms of chemical elements, during which an increase in the total number of electrons in an atom is accompanied by an increase in the number of electron shells with different values of the main and orbital quantum numbers.

\subsection{Function of structural development}

For a quantitative assessment of the structural development of systems, it seems natural to use the values of a certain function, the arguments of which are the additive syntropy $I_{\Sigma}$ and the entropy of reflection $S$. The behavior of such a function must be made consistent with the general laws of structural development, which can be represented as follows.

When $N=1$, all elements of the system have the same distinguishing feature, the division of the system into parts is absent, and the structure of the system is not developed. In this case, $I_{\Sigma}=I_{A}, S=0$. In the interval $1<N<|A|$, the formation takes place of various groups of elements with the same values of the attribute $P_{B}=P_{B_{1}}, \ldots, P_{B_{N}}$, as a result of which the system is divided into $N$ parts and its structural development occurs, for which, $0<I_{\Sigma}<I_{A}, 0<S<I_{A}$. In the state of the system, when $N=|A|$, each element has an individual value of the attribute $P_{B}$, and the system is split up into parts consisting of only one element. This corresponds to the complete destruction of any structure, that is, in this state of the system there is no structural development and $I_{\Sigma}=0, S=I_{A}$.

It follows from the above that when a system moves from the state $N=1$ to the state $N=|A|$, the values of the function of structural development must first increase from zero to a certain maximum, and then decrease again to zero. As such a function, it is natural to take the product $I_{\Sigma} \cdot S$, which we will call the D-function of the structural development of discrete systems ( $D$ is the first letter of the word development):

$$
D=I_{\Sigma} \cdot S
$$

The structural development function (61) assumes the minimum value $D^{\text {min }}=0$ when $N=1$ and $N=|A|$, whereas this function has the maximum value $D^{\max }$ when $I_{\Sigma}=S=I_{A} / 2$. The latter is explained by the fact that the product of two non-negative numbers with a constant sum is maximal when the numbers are equal. That is, the development function (61) is characterized by the fact that its values are the greater, the smaller the absolute value $\left|I_{\Sigma}-S\right|$ is, and, accordingly, we have:

$$
D^{\max }=\frac{I_{A}^{2}}{4} .
$$

In other words, the system reaches the maximum possible level of development when chaos and order in its structure are equal to each other $(R=1)$.

In the general case, systems with different numbers of elements may have the same value of the development function $D$. In this case, if, for example, two systems $A_{1}$ and $A_{2}$ are given such 
that $\left|A_{1}\right|<\left|A_{2}\right|$ and $D_{A_{1}}=D_{A_{2}}$, then it is clearly understood that the system $A_{1}$ is at a higher level of development than the system $A_{2}$. Therefore, when comparing the structural development of various systems, it is expedient to use the relative value of development $D^{*}$, which is convenient to express as a percentage:

$$
D^{*}=\frac{D}{D^{\max }}=\frac{4 D}{I_{A}^{2}}, \% .
$$

In order to have a visual representation of the behavior of the structural development function, Table 5 shows its absolute and relative values, as well as the values $I_{\Sigma}, S, R$, for different states of the discrete system in Figure 7.

Table 5. Informational characteristics of the system states in Figure 6.

\begin{tabular}{clcccccc}
\hline $\begin{array}{c}\text { State of the } \\
\text { system }\end{array}$ & Type of the system & $\boldsymbol{N}$ & $\boldsymbol{I}_{\Sigma}$ & $\boldsymbol{S}$ & $\boldsymbol{R}$ & $\boldsymbol{D}$ & $\boldsymbol{D}^{*}$ \\
\hline (a) & Orderly & 1 & 4 & 0 & $\infty$ & 0 & $0 \%$ \\
(b) & Orderly & 2 & 3 & 1 & 3 & 3 & $75 \%$ \\
(c) & Harmonious & 4 & 2 & 2 & 1 & 4 & $100 \%$ \\
(d) & Chaotic & 8 & 1 & 3 & 0.33 & 3 & $75 \%$ \\
(e) & Chaotic & 16 & 0 & 4 & 0 & 0 & $0 \%$ \\
\hline
\end{tabular}

A joint analysis of (32)-(35) and (61) suggests that with all the variety of possible variants of the structural development of systems, as they move from the state $N=1$ to the state $N=|A|$, there are two polarly opposite variants when development goes along fundamentally different ways. The first of these variants is characterized by the fact that for each $N$ the entropy of reflection $S$ takes its maximum value (32) and the development follows the path of maximum possible structural chaos. We will call this variant the entropic variant of the development of systems, whereas we denote the corresponding values of $D$-function by the symbol $D_{S}$, that is:

$$
D_{S}=S^{\max } \cdot I_{\Sigma}^{\min }
$$

In the second variant of development, we have the opposite situation, when for any value of $N$ already the additive syntropy $I_{\Sigma}$ assumes the maximum value (34) and development follows the path of maximum possible orderliness. By analogy with the first variant, we will call this development path of the systems the syntropic variant and denote the corresponding values of $D$ function by the symbol $D_{I}$, that is:

$$
D_{I}=I_{\Sigma}^{\max } \cdot S^{\min }
$$

Let us now consider the nature of the change in the values of $D$-function for the intensive and extensive types of development of systems.

\subsection{Features of the intensive type of system development}

Let us construct the graphs of the dependence of $D_{S}, D_{I}, D^{\max }$ on $N$ for $|A|=$ const and analyze the resulting diagram, which is shown in Figure 11. 


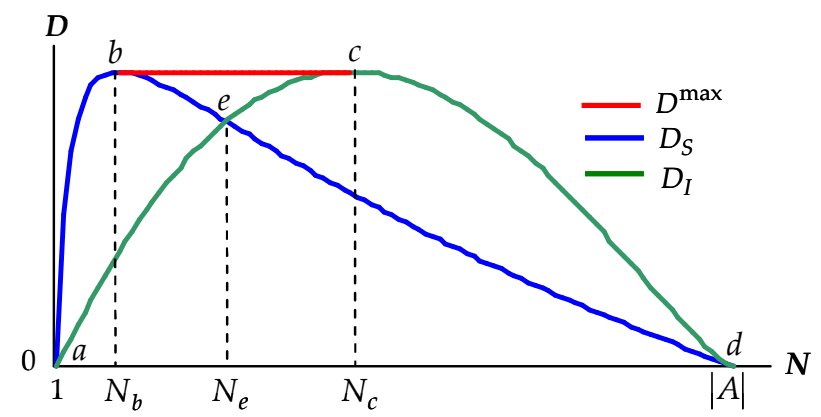

Figure 11. Graphs of structural development functions for the intensive type of system development $(|A|=100)$.

It can be seen from the figure that the range of possible values of the development function (61) for the intensive type of development of systems is limited by the diagram contour abcdea. The points $b$ and $c$, at which $D_{s}=D^{\max }$ and $D_{I}=D^{\max }$, correspond to the points $b$ and $f$ of the information field of reflection in Figure 8, which allows us to identify, on the basis of projections of these points, the left $\left(1 \leq N<N_{b}\right)$, central $\left(N_{b} \leq N \leq N_{c}\right)$ and right $\left(N_{c}<N \leq|A|\right)$ intervals of the values of $N$, already known to us, on the horizontal axis. In each of these intervals, the structural development of systems has its own features, which are as follows.

In the left interval of order $D_{S}>D_{I}$ and as $N$ increases, there is observed a general trend of increasing of the $D$-function values, which corresponds to the progressive nature of the intensive type of structural development of systems. In this case, those systems that develop in the entropy variant (along the $a b$ line) have the highest level of development. And, on the contrary, those systems, the development of which takes place according to the syntropic variant (along the ae line), are least developed. That is, in the left interval, for a specific value of $N$, an increase in the entropy $S$ is a positive factor in the development of the system, whereas an increase in syntropy $I_{\Sigma}$ is a negative factor.

In the right interval of chaos $D_{I}>D_{S}$ and with an increase in the values of $N$, there occurs a general decrease in the values of $D$-function, which testifies to structural degradation of systems. In this case, the most developed are those systems whose development follows the syntropic variant (along the $c d$ line). Accordingly, the systems developing along the entropy variant (along the $e d$ line) have a minimum level of development in the right interval. That is, in the right interval, for a specific value of $N$, a positive factor in the development of systems is an increase in syntropy $I_{\Sigma}$ and a decrease in entropy $S$.

In the central interval of harmony, various situations are possible, when $D_{S}>D_{I}$ or $D_{S}<D_{I}$. Accordingly, there is observed a more complex picture here of the structural development of systems than in the left and right intervals. In this case, on the diagram at the point $e$, equality $D_{I}=D_{S}$ is observed, while the projection $N_{e}$ of this point on the horizontal axis divides the central interval into the left $\left(N_{b} \leq N \leq N_{e}\right)$ and right $\left(N_{e}<N \leq N_{c}\right)$ parts, in each of which the development of systems has its own features. These features are reduced to the fact that in the left part there is a tendency of the values $D_{S}$ to exceed the values $D_{I}$, which fades as $N$ increases; while in the right part there takes place an opposite tendency, which only grows with increasing $N$. In addition, throughout the central interval, the inequalities $D \geq D_{S}$ and $D \geq D_{I}$ can be fulfilled simultaneously, and, accordingly, the system can reach its maximum level of development at which $D=D^{\max }$ (along the $b c$ line). All this suggests that in the central interval of the values of $N$, there is observed a general tendency towards the harmony of interrelations between various options for the development of systems.

Thus, it follows from the above that, for the intensive type of development, in the process of increasing the number of parts of the system from $N=1$ to $N=|A|$, the system in its structural 
development consistently passes through three stages: the progress stage, the harmony stage and the degradation or regression stage.

\subsection{Features of extensive type of system development}

Figure 12(a) shows the graphs of dependence of the values of $D_{S}, D_{I}, D^{\max }$ on $|A|$ for the extensive type of development of systems, and since for this type $D^{\max } \neq$ const, Figure $12(\mathrm{~b})$ also shows the graphs of the relative values $D_{S}^{*}, D_{I}^{*}, D^{\text {max* }}$. Let us consider the features of extensive development that follow from these graphs.

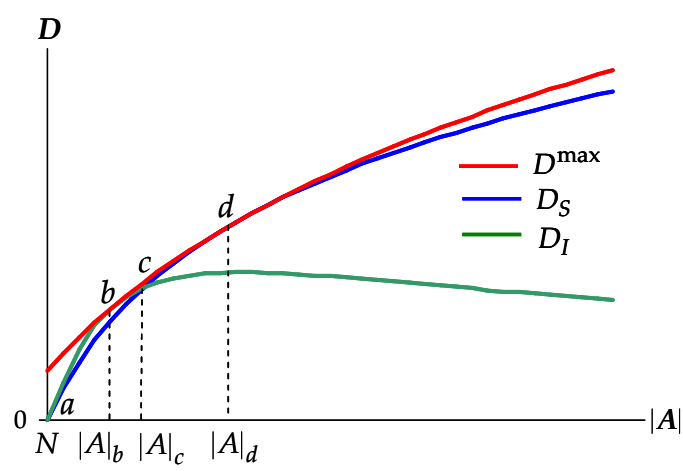

(a)

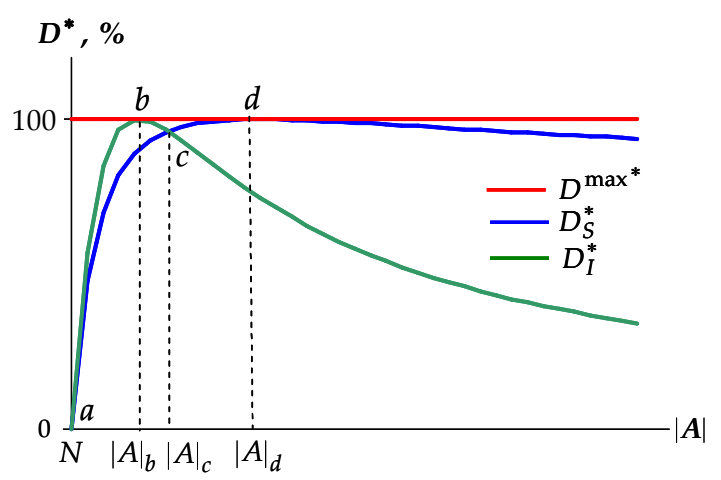

(b)

Figure 12. Graphs of functions of structural development for the extensive type of development of systems $(N=4)$ : (a) graphs of $D_{S}, D_{I}, D^{\max } ;(\mathbf{b})$ graphs of $D_{S}^{*}, D_{I}^{*}, D^{\text {max* }}$.

First of all, we note that the points $a, b, c, d$ in both figures correspond to the same values of $|A|$. In this case, at the points $b$ and $d$ in Figure $12(\mathrm{a})$, the values $D_{I}=D^{\text {max }}$ and $D_{S}=D^{\text {max }}$ are observed, respectively, whereas in Figure $12(\mathrm{~b}), D_{I}^{*}=100 \%$ and $D_{S}^{*}=100 \%$. That is, these points correspond to points $f$ and $b$ of the reflection information field in Figure 8. This circumstance allows, on the basis of the projections of points $b$ and $d$, to distinguish three intervals of values of $|A|$ and, depending on the ratio of $|A|$ and $N$, to put into correspondence to these intervals the already known intervals of the values of $N$ for $|A|=$ const. We show these intervals and indicate the peculiarities of behavior within their limits of the functions $D_{S}, D_{I}, D_{S}^{*}, D_{I}^{*}$.

The first interval $N \leq|A|<|A|_{b}$ corresponds to the right interval of chaos, and the more developed here are the systems whose development follows the syntropic variant. That is, all the specified functions in the first interval increase their values and the inequalities $D_{I}>D_{S}$ and $D_{I}^{*}>D_{S}^{*}$ are satisfied.

The third interval $|A|_{d}<|A| \leq \infty$ is similar to the left interval of order in terms of the ratio of the values of $|A|$ and $N$, and those systems in this interval have a higher level of development that evolve according to the entropy variant. That is, in comparison with the first interval, the opposite inequalities $D_{I}<D_{S}$ and $D_{I}^{*}<D_{S}^{*}$ are always satisfied there, but at the same time, while, for the syntropic variant of development, the values of $D_{I}$ and $D_{I}^{*}$ decrease all the time and asymptotically approach zero as $|A| \rightarrow \infty$, then, for the entropic variant, the values of $D_{S}$ increase and the values of $D_{S}^{*}$ gradually decrease.

The second interval $|A|_{b} \leq|A| \leq|A|_{d}$ corresponds to the central interval of harmony and various situations are possible in it, when $D_{I}>D_{S}$ or $D_{I}<D_{S}$, and also $D_{I}^{*}>D_{S}^{*}$ or $D_{I}^{*}<D_{S}^{*}$. In this case, from the beginning to the end of the interval, the functions $D_{S}, D_{I}, D_{S}^{*}$ increase their 
values, while the values of $D_{I}^{*}$ are constantly decreasing. Note also that, by the projection of the point $c$, at which $D_{I}=D_{S}, D_{I}^{*}=D_{S}^{*}$, the second interval is further divided into left $\left(|A|_{b} \leq|A| \leq|A|_{\mathcal{C}}\right)$ and right $\left(|A|_{\mathcal{C}}<|A| \leq|A|_{d}\right)$ parts. In the first of these parts $D_{I}>D_{S}$ and $D_{I}^{*}>D_{S}^{*}$, that is, more developed here are the systems, the evolution of which follows the syntropic variant. In the second part, in turn, $D_{I}<D_{S}$ and $D_{I}^{*}<D_{S}^{*}$, that is, a higher level of development in this part have systems that develop according to the entropic variant. In this case, in both parts the relative level of development of systems can reach its maximum value $D^{*}=100 \%$.

Thus, what has been said indicates that in the process of extensive development, according to the ratio of values $|A|$ and $N$, the system passes through the intervals of the values of $N$ in the reverse order compared to the intensive type of development. That is, in the case of extensive development, the system is first in the right interval of chaos, then passes through the central interval of harmony and enters the left interval of order where it remains during all subsequent time for $|A| \rightarrow \infty$.

\section{Examples of using STI}

It is obvious that the informational-synergetic functions $I_{A}, I_{A B}, I_{\Sigma}, S, R, D, S, R, D$ can be used in the analysis of discrete systems of any nature. Let us demonstrate this, using simple examples in such distant from each other fields as medicine and linguistics.

\subsection{Assessment of the interconnection between diseases}

In modern clinical medicine, much attention is paid to analyzing the phenomena of comorbidity, that is, the presence of several diseases in a patient at the same time. In this case, of the real significance is the quantitative assessment of the interconnection between various pairs of diseases, which can be performed using the average value of the relative syntropy of reflection (22). In this case, the assessment of the interconnection between two diseases $A$ and $B$ and its further use are as follows.

Suppose that among a representative set of people with a particular disease (system $D$ ), three groups of patients are distinguished. The first group is people with the disease $A$ (set $A$ with the number of elements $|A|$ ). The second group is people with the disease $B$ (set $B$ with the number of elements $|B|$ ). The third group consists of people who have both diseases $A$ and $B$ at the same time (set $K=A \cap B$ with the number of elements $|K|$ ). By first determining the value of the syntropy of reflection (16), and then, by calculating its average relative value (22), we will get an answer to the question concerning the extent to which diseases $A$ and $B$ are interconnected with each other. Accordingly, when diagnosing a person with one disease, we can, with a certain degree of confidence, predict the presence or risk of the other disease. Also with the help of this assessment it is possible to conduct a comparative analysis of the interconnection of the same diseases in different groups of patients, distinguished on the basis of gender, age, area of residence, etc. We show this on a specific calculation example.

In [20], on the basis of an analysis of autopsies in one of large hospitals, the following data are presented on such diseases as coronary heart disease (CHD, set $A$ ) and diseases of the urinary system (DUS, set $B$ ). Men: CHD - 807 people, DUS - 752 people; women: CHD - 1366 people, DUS - 1419 people. At the same time, both CHD and DUS were observed in men 458 times, and in women, 867 times. Conducting calculations using formulas (16) and (22), we obtain the following results:

$I_{A B}(\operatorname{man})=\frac{458^{2}}{807 \cdot 752} \log _{2} 458=3.055, \quad I_{A B}^{*}($ man $)=\frac{3.055}{2}\left(\frac{1}{\log _{2} 807}+\frac{1}{\log _{2} 752}\right)=0.318$, 


$$
I_{A B}(\text { woman })=\frac{867^{2}}{1366 \cdot 1419} \log _{2} 867=3.785, \quad I_{A B}^{*}(\text { woman })=\frac{3.785}{2}\left(\frac{1}{\log _{2} 1366}+\frac{1}{\log _{2} 1419}\right)=0.362
$$

The obtained results suggest that the interconnection between coronary artery disease and diseases of the urinary system in women is stronger than in men.

\subsection{Analysis of texts}

Any text can be represented as a discrete system $A$, whose elements are the letters of the alphabet used. In this case, the sets of identical letters form parts $B_{1}, \ldots, B_{N}$ of the system, whose number and mutual relations in terms of the number of elements determine the overall structure of the text. Accordingly, using the STI, it is possible to assess the structural organization (58) and the level of development (61), (63) of an arbitrary text, as well as to compare any texts according to these indicators, regardless of the language in which they are written. Let us give an example of such a comparison, for which we take the well-known words of Shakespeare's Hamlet written in English, French, German and Spanish:

- English: to be, or not to be, that is the question;

- French: etre ou ne pas être: telle est la question;

- German: sein oder Nichtsein, das ist hier die Frage;

- Spanish: ser o no ser, esa es la cuestión.

For each variant of writing Table 6 shows the frequencies of occurrence of individual letters $\left(\left|B_{i}\right|\right)$, whereas Table 7 gives the values of information-synergetic functions, calculated for these frequencies, characterizing the structural organization $(R)$ and the level of development $(D)$ of Shakespeare's phrase in terms of ratio of chaos $(S)$ and order $\left(I_{\Sigma}\right)$ in its structure.

Table 6. The frequency of occurrence of letters in the phrase "To be, or not to be, that is the question".

\begin{tabular}{|c|c|c|c|c|}
\hline \multirow[b]{2}{*}{ Letter } & \multicolumn{4}{|c|}{ Language } \\
\hline & 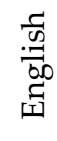 & 苞 & 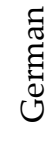 & 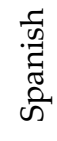 \\
\hline Space & 9 & 8 & 7 & 7 \\
\hline$a$ & 1 & 2 & 2 & 2 \\
\hline$b$ & 2 & 0 & 0 & 0 \\
\hline c & 0 & 0 & 1 & 1 \\
\hline$d$ & 0 & 0 & 3 & 0 \\
\hline$e$ & 4 & 9 & 6 & 5 \\
\hline$f$ & 0 & 0 & 1 & 0 \\
\hline$g$ & 0 & 0 & 1 & 0 \\
\hline$h$ & 2 & 0 & 2 & 0 \\
\hline$i$ & 2 & 1 & 6 & 1 \\
\hline$j$ & 0 & 0 & 0 & 0 \\
\hline$k$ & 0 & 0 & 0 & 0 \\
\hline$l$ & 0 & 3 & 0 & 1 \\
\hline$m$ & 0 & 0 & 0 & 0 \\
\hline$n$ & 2 & 2 & 3 & 2 \\
\hline$o$ & 5 & 2 & 1 & 3 \\
\hline$p$ & 0 & 1 & 0 & 0 \\
\hline$q$ & 1 & 1 & 0 & 0 \\
\hline$r$ & 1 & 2 & 3 & 2 \\
\hline$s$ & 2 & 3 & 4 & 5 \\
\hline$t$ & 7 & 5 & 2 & 1 \\
\hline
\end{tabular}




\begin{tabular}{ccccc}
$u$ & 1 & 2 & 0 & 1 \\
$v$ & 0 & 0 & 0 & 0 \\
$w$ & 0 & 0 & 0 & 0 \\
$x$ & 0 & 0 & 0 & 0 \\
$y$ & 0 & 0 & 0 & 0 \\
$z$ & 0 & 0 & 0 & 0 \\
\hline
\end{tabular}

Table 7. Informational characteristics of the phrase "To be, or not to be, that is the question".

\begin{tabular}{ccccccccc}
\hline Language & $|\boldsymbol{A}|$ & $\boldsymbol{N}$ & $\boldsymbol{I}_{\boldsymbol{A}}$ & $\boldsymbol{I}_{\boldsymbol{\Sigma}}$ & $\boldsymbol{S}$ & $\boldsymbol{R}$ & $\boldsymbol{D}$ & $\boldsymbol{D}^{*}$ \\
\hline English & 39 & 13 & 5.286 & 1.995 & 3.291 & 0.606 & 6.566 & $94.0 \%$ \\
French & 41 & 13 & 5.358 & 2.041 & 3.317 & 0.615 & 6.770 & $94.3 \%$ \\
German & 42 & 14 & 5.392 & 1.879 & 3.513 & 0.535 & 6.601 & $90.8 \%$ \\
Spanish & 31 & 12 & 4.954 & 1.730 & 3.224 & 0.537 & 5.578 & $90.9 \%$ \\
\hline
\end{tabular}

Analysis of Table 7 allows making the following observations. All spellings of the Shakespeare phrase in different languages are clearly divided into two groups. The first group consists of English and French versions, which differ slightly from each other in the values of the functions $R$ and $D^{*}$. In this case, the difference between the values of $R$ is 0.009 with the average value 0.61 for the group, whereas the difference between the values of $D^{*}$ is equal to $0.3 \%$ with the average value of $94.15 \%$. The second group includes the German and Spanish versions, which practically do not differ from each other in terms of the values of $R$ and $D^{*}$. The difference between the values of $R$ in these variants is only 0.002 with the average value of 0.536 , while the difference in values of $D^{*}$ equals $0.1 \%$ with the average value of $90.85 \%$. That is, the difference between the group average values exceeds the average difference of values within the groups by a factor of $\frac{2(0.61-0.536)}{0.009+0.002}=13.45$ with respect to $R$-function, and by $\frac{2(94.15 \%-90.85 \%)}{0.3 \%+0.1 \%}=16.5$ times, with respect to the relative value of $D$-function. This suggests that the text of the Shakespeare's phrase, written in English and French, differs significantly in its structural organization and level of development from the text written in German and Spanish.

\subsection{Characteristic length of the text}

In the given example with the Shakespeare's phrase written in different languages, one and the same alphabet with $n=27$ letters, including the space, was used, whereas the average text length according to Table 7 was $|\bar{A}|=38.25$ letters with the average $\bar{N}=13$ letters used. Moreover, in all cases the additive syntropy $I_{\Sigma}$ turned out to be less than the entropy of reflection $S$. At the same time, if we begin to evaluate the structural organization and development of larger fragments of the Shakespearean play or even the entire play as a whole, then, since $N \leq n$, we will inevitably come to that the entropy $S$ begins to oscillate around a certain constant value $S_{g}$, conditioned by the grammatical structure of the language, while syntropy $I_{\Sigma}$ will steadily increase. In other words, if $|A| \rightarrow \infty$, then the inequality $I_{\Sigma}>S$ is always fulfilled, and the change in the values of $R$ and $D$ depends only on the orderliness of the text, while the randomness of the text does not actually play any role. This suggests that, while performing a comparative analysis of the texts with respect to the ratio in their structure of chaos and order, it is advisable to impose certain restrictions on the length of the estimated sections in such a way that entropy $S$ can influence the value of $R$ and $D$ to the same extent as syntropy $I_{\Sigma}$. That is, it is necessary to establish such a maximum length of the analyzed text so that when using all the letters of the alphabet $(N=n)$, equality $S_{g}=I_{\Sigma}$ can be fulfilled. We will call this length the characteristic length of the text and denote it by the symbol $\lambda$. The value of $\lambda$ is determined based on the fact that when $|A|=\lambda$ there takes place the equality $I_{A}=\log _{2} \lambda=2 S_{g}$. It follows that 


$$
\lambda=4^{S_{g}} .
$$

The quantity $S_{g}$ depends on the probability of appearance in the text of various letters of the alphabet used, and, since $S=H$, in calculating the values of $\lambda$ by the formula (66), Shannon's entropy can be used, which is currently defined for many languages. An example of such a calculation is presented in Table 8, where the values of $H$ [21] and $\lambda$ are given for English, French, German, and Spanish.

Table 8. Entropy $H$ and the characteristic text length $\lambda$ for various languages.

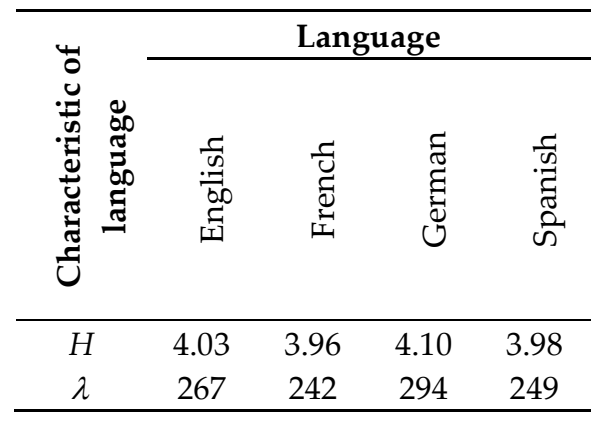

Naturally, when characterizing and comparing large texts, they should first be fragmented into sections with the number of letters $|A| \leq \lambda$, and then the average values of the corresponding information-synergetic functions should be determined. As follows from Table 8 , the fragments of text written in German may have the greatest length.

\section{Conclusion}

In the presented material, on the basis of simple axioms, without reliance on probability theory and traditional information theory, the path has been traveled from considering the information aspects of reflecting a single finite set to evaluating the structural organization and the level of development of discrete systems. In addition, a new approach to defining the amount of information was developed, in which information is understood as the data about a finite set as a single whole, and previously unknown information-synergetic functions were obtained that have an integrative-code connection with the Hartley and Shannon information measures. In addition, three information laws have been established: the law of conservation of the sum of chaos and order, the information law of reflection and the law of conservation and transformation of information. All this suggests that the synergetic theory of information is a new direction of information-theoretical research. Further development of this theory and its introduction into the practice of scientific and applied research, in our opinion, will be important not only for the general theory of information, but also for those subject areas in which the objects of cognition are discrete systems with a finite set of elements.

Conflicts of Interest: The author declare no conflict of interest.

\section{Appendix A. Asymmetry of the finite set reflection and commensurability of arbitrary quantities. Syntropic and golden ratios.}

\section{A1. Asymmetry of reflection and commensurability of finite sets}

It was noted in Section 2.3 that the difference between the finite sets $A$ and $B$ in the number of elements determines the asymmetry of the completeness of their reflection through each other. Let us analyze this asymmetry; to that end, we represent its equation (21) in the expanded form: 


$$
A s=\frac{|K|^{2}}{|A| \cdot|B|} \cdot \log _{2}|K| \cdot\left|\frac{1}{\log _{2}|A|}-\frac{1}{\log _{2}|B|}\right| .
$$

Equation (A1) refers to the most general case of interrelations of sets $A, B, K$ and simply shows that $A s>0$, if $|A| \neq|B|$ and $|K|>1$. In more particular cases, when one of the sets $A$ or $B$ is a subset of another set, that is, when either $B \subset A$ or $A \subset B$ and, respectively, $|K|=|B|$ or $|K|=|A|$, equation (A1) assumes the form:

$$
\begin{aligned}
& A s_{B \subset A}=\frac{|B|}{|A|}\left(1-\frac{\log _{2}|B|}{\log _{2}|A|}\right), \\
& A s_{A \subset B}=\frac{|A|}{|B|}\left(1-\frac{\log _{2}|A|}{\log _{2}|B|}\right) .
\end{aligned}
$$

Let us consider the specificities of asymmetry As, arising from equations (A2) and (A3). We first note that for $|B|=1$ equation (A2) produces a paradoxical at first glance situation, which consists in the following. When $|B|=1$, then, according to the syntropy equation (16), sets $A$ and $B$ do not reflect any information about each other $\left(I_{A B}=0\right)$, so that equality $A s=0$ must hold. At the same time, the asymmetry equation (A2) shows that, if $|B|=1$, then $A s=1 /|A|$. This paradox is explained by the fact that, when $B \subset A$, then in the equation of relative syntropy (20), logarithms are cancelled out and it assumes the form $I_{B \rightarrow \mathrm{A}}^{*}=|B| /|A|$. Accordingly, for $|B|=1$, an illusory equality $I_{B \rightarrow \mathrm{A}}^{*}=1 /|A|$ is created. Equation (A2) takes this equality into account and shows that, for $|B|=1$, the asymmetry $A s$ is supposedly different from zero. That is, this paradox has a formal illusory character, and when $|A|>>1$ it practically disappears, because, if $|A| \rightarrow \infty$, then $A s=1 /|A| \rightarrow 0$. Therefore, in the sequel, we will not pay attention to this paradox, assuming that, if $|B|=1$, then this is a limiting degenerate case.

Figure A1 presents the graphs of the dependence $A$ s on $|B|$, calculated from equations (A2) and (A3) for $|A|=$ const .

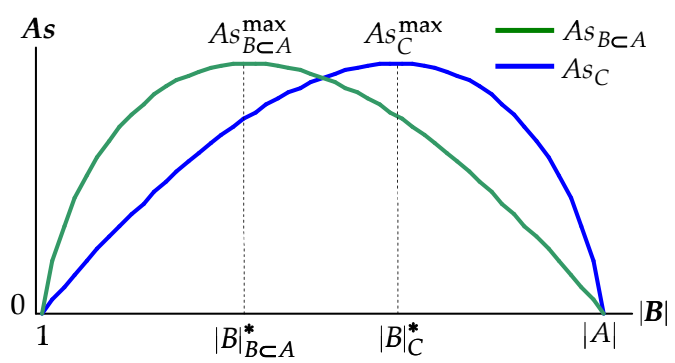

(a)

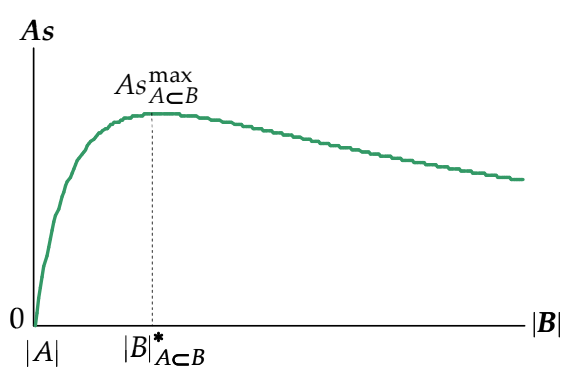

(b)

Figure A1. The dependence of $A s_{B \subset A}, A s_{A \subset B}, A s_{C}$ on $|B|$ for $|A|=$ const: (a) $B \subset A$; (b) $A \subset B$.

It can be seen from the figure that asymmetry As has the same character of the change in its values, regardless of whether the set $B$ is a subset (Figure A1(a)) or a superset (Figure A1(b)) of the set $A$. That is, for $B \subset A$ and when the values of $|B|$ increase, the asymmetry first increases from $A s=0$ to the maximum value $A s_{B \subset A}^{\max }$ at $|B|_{B \subset A}^{*}$, then decreases and for $|B|=|A|$ it reaches the 
value $A s=0$ again. Similarly, when $A \subset B$, the asymmetry first increases to the value $A s_{A \subset B}^{\max }$ at $|B|=|B|_{A \subset B}^{*}$, and then decreases to the value $A s \approx 0$ for $|B|>>|A|$.

It is obvious that such behavior of asymmetry is related to the commensurability of sets, that is, to their correspondence to each other in the number of elements. Indeed, the sets $A$ and $B$ are commensurate to each other to the maximum extent when $|B|=|A|$, and to the same degree are incommensurable when either $|B|=1$ or $|B|>|A|$. In all these cases, the asymmetry has minimal values. This suggests that on the set of values $|B|=1,2, \ldots$ for any $|A|=$ const there is an interval on which the sets $A$ and $B$ are more commensurable to each other than beyond its boundaries. It is natural to assume that these boundaries are the values $|B|_{B \subset A}^{*}$ and $|B|_{A \subset B}^{*}$, for which the asymmetry has its maximum values $A s_{B \subset A}^{\max }$ and $A s_{A \subset B}^{\max }$. In other words, the sets $A$ and $B$ are commensurable in the number of elements if the following inequality holds:

$$
|B|_{B \subset A}^{*} \leq|B| \leq|B|_{A \subset B}^{*} .
$$

Let us represent inequality (A4) in expanded form; to this end, we find approximately the extrema of the functions of $|B|$ in the right-hand sides of relations (A2) and (A3). In this case, the results obtained will be rounded to the nearest larger integer, since the number of elements in the composition of a finite set can be expressed only by a natural number.

After differentiating the right-hand side of (A2), we obtain the equation

$$
\ln |B|-\ln |A|+1=0,
$$

solving which yields

$$
|B|_{B \subset A}^{*}=\left\lceil\frac{|A|}{e}\right\rceil
$$

where $e=2.71828 \ldots$ is the base of natural logarithms.

The analogous differentiation of the right-hand side of (A3), in turn, leads to the equation

$$
(\ln |B|)^{2}-\ln |A| \cdot \ln |B|-\ln |A|=0,
$$

solving which, we get

$$
|B|_{A \subset B}^{*}=\left\lceil\exp \frac{1}{2}\left(\ln |A|+\sqrt{(\ln |A|)^{2}+4 \ln |A|}\right)\right\rceil .
$$

Substituting the values $|B|_{B \subset A}^{*}$ and $|B|_{A \subset B}^{*}$ from (A5) and (A6) into inequality (A4), we obtain the interval of commensurability of finite sets $A$ and $B$ with respect to the number of elements:

$$
\left\lceil\frac{|A|}{e}\right\rceil \leq|B| \leq\left\lceil\exp \frac{1}{2}\left(\ln |A|+\sqrt{(\ln |A|)^{2}+4 \ln |A|}\right)\right\rceil .
$$

In addition, if $B \subset A$, then the interval of commensurability (A7) takes the form:

$$
\left\lceil\frac{|A|}{e}\right\rceil \leq|B| \leq|A|
$$

Let us consider the situation $B \subset A$ in more detail. In this case, one more subset $C=A \backslash B$ with the number of elements $|C|=|A|-|B|$ is identified in the set $A$. The equation for the asymmetry $A s_{C}$ of the reflection of this subset through the set $A$ is analogous to equation (A2) and has the form: 


$$
A s_{C}=\left(1-\frac{|B|}{|A|}\right) \cdot\left(1-\frac{\log _{2}(|A|-|B|}{\log _{2}|A|}\right) .
$$

Figure A1(a) shows the graph of the dependence $A s_{C}$ on $|B|$, calculated from equation (A9). The figure shows that the graph of $A s_{C}$ is mirror-symmetric to the graph of $A s_{B \subset A}$ and has the maximum $A s_{C}^{\max }$ when $|B|=|B|_{C}^{*}$. Let us determine the value of $|B|_{C}^{*}$; to this end, we equate to zero the derivative with respect to $|B|$ of the right-hand side of (A9). As a result, we have the equation

$$
\ln |A|-\ln (|A|-|B|)-1=0,
$$

solving which, we have:

$$
|B|_{C}^{*}=\left[\left(1-\frac{1}{e}\right) \cdot|A|\right]
$$

A joint analysis of equations (A5) and (A10), taking into account inequality (A8), allows us to state the following. When $|B|<|B|_{A \subset B}^{*}$, the subset $B$ is not commensurate with the set $A$, while the subset $C$ is commensurate. In contrast, if $|B|>|B|_{C}^{*}$, then the subset $C$ is incommensurable, whereas the subset $B$ is commensurate with the set $A$. It follows that the set $A$ can be divided into two commensurate with it subsets $B$ and $C$ when the following inequality holds:

$$
\left\lceil\frac{|A|}{e}\right\rceil \leq|B| \leq\left\lceil\left(1-\frac{1}{e}\right) \cdot|A|\right\rceil \text {. }
$$

In this case, since $|B|=|A|-|C|$, in accordance with the expression (A8) and when the inequality (A11) is satisfied, the subsets $B$ and $C$ are commensurate not only with the set $A$, but also with each other.

\section{A2. Commensurability of arbitrary quantities}

Inequalities (A8) and (A11) are obtained on the basis of information analysis of the reflection of intersecting finite sets $A$ and $B$ through each other, the number of elements in the composition of which is a natural number. At the same time, it seems natural to adapt these inequalities to analyze the commensurability of arbitrary quantities $a, b$ and $c=a-b$. In this case, inequality (A8) is transformed into an interval of commensurability of two quantities $a$ and $b \leq a$ :

$$
\frac{a}{e} \leq b \leq a .
$$

Inequality (A11), in turn, is transformed into an interval of commensurability of the quantity $a$ with each of its parts $b$ and $c=a-b$ :

$$
\frac{a}{e} \leq b \leq\left(1-\frac{1}{e}\right) a .
$$

In this case, as in the case of finite sets, when inequality (A13) is satisfied, the parts $b$ and $c=a-b$ are commensurate to each other.

When the whole and its parts are commensurate with each other, we say that they are in a harmonious relation. In accordance with this, the division of the quantity $a$ into two parts in the interval (A13) will be called a harmonic division. In this case, the extreme such divisions at the ends of the interval will be named, in general, a "syntropic ratio" (at the left end of the interval, the left syntropic ratio $1 / e$; at the right end of the interval, the right syntropic ratio $\frac{e-1}{e}$ ). That is, the syntropic ratio is the extreme case of a harmonious division of an arbitrary quantity into two parts commensurate with it. This name for the extreme harmonious divisions is due to the fact that they are 
genetically related to the maximum value of the difference in the relative values of the reflection syntropy of finite sets.

The left and right syntropic ratios simultaneously divide an arbitrary quantity $a$ into three parts $b_{1}, b_{2}, b_{3}$, such that $b_{1}=b_{3}=\frac{a}{e}, b_{2}=\frac{a(e-2)}{e}$, or in a rounded percentage expression, $b_{1}=b_{3}=37 \%, b_{2}=26 \%$. This division is shown graphically in Figure A2, where a segment of a unit length $(a=1)$ is divided by the syntropic ratios.

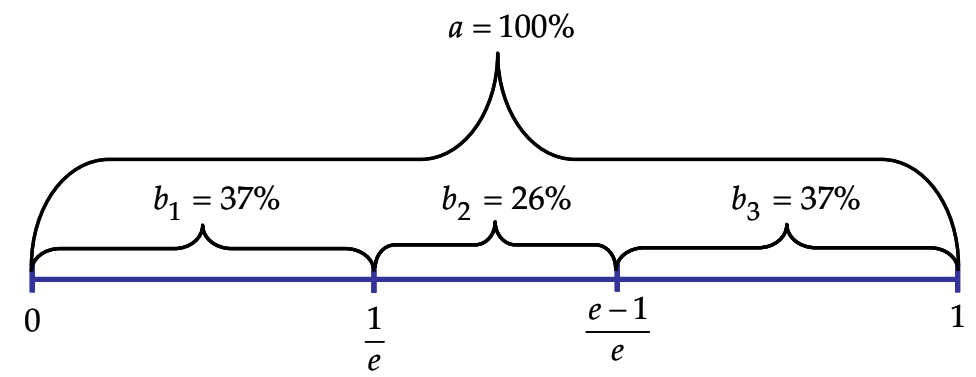

Figure A2. Division of the segment $a=1$ by syntropic ratios.

In this case, the parts $b_{1}$ and $b_{3}$ are commensurate with the quantity $a$, whereas the part $b_{2}$ is incommensurable with this quantity because of the inequality $\frac{a(e-2)}{e}<\frac{a}{e}$. It follows that an arbitrary quantity cannot be divided into more than two parts commensurate with it.

\section{A3. Triadic chain}

It follows directly from the syntropic ratio $1 / e$ that the number $e$ is divided by this section into two parts 1 and $e-1$. In this case, the number $e$ itself is one of the two parts $e$ and $e(e-1)$, into which, in turn, the number $e^{2}$ is also divided by the syntropic ratio; and so on. This indicates that each term of a geometric progression $e^{1}, e^{2}, e^{3}, \ldots$ is divided into two parts by the syntropic ratio and together with these parts forms a triad $T_{k}=\left\langle e^{k-1},(e-1) e^{k-1}, e^{k}\right\rangle, k=1,2, \ldots$. The union of such triads $\bigcup_{k=1}^{\infty} T_{k}$, in turn, forms an increasing sequence

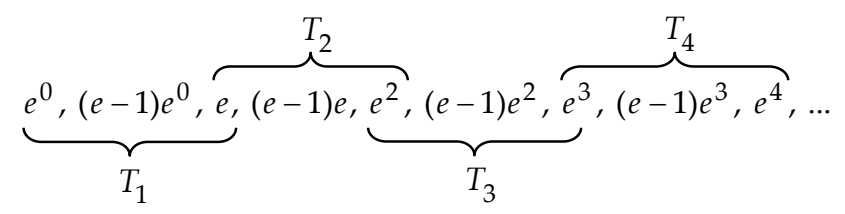

the even and odd members of which are equal to: $a_{2 n}=e^{n}, a_{2 n+1}=(e-1) e^{n}, n=0,1,2, \ldots$. In this case, any member of the sequence $a_{n}, n>0$ is commensurate with each of its neighboring members $a_{n-1}$ and $a_{n+1}$, whereas the ratio of the two triads $T_{k}$ and $T_{k+1}$ with respect to the sum of its members is equal to the syntropic ratio, that is:

$$
\frac{\left|T_{k}\right|}{\left|T_{k+1}\right|}=\frac{e^{k-1}+(e-1) e^{k-1}+e^{k}}{e^{k}+(e-1) e^{k}+e^{k+1}}=\frac{1}{e} .
$$

The intersection of the triads $T_{k} \cap T_{k+1}=e^{k}$ indicates that the sequence (A14) has a chain structure, the links of which are the triads $\left\{T_{k}\right\}$. Therefore, we will call this sequence a triad chain.

All the members of the triad chain (A14) for $n>0$ are irrational numbers. Rounding these numbers to a near larger integer converts the triad chain into a sequence $\left\{T_{k}^{*}\right\}$, 
$T_{k}^{*}=\left\langle\left\lceil e^{k-1}\right\rceil,\left\lceil(e-1) e^{k-1}\right\rceil,\left\lceil e^{k}\right\rceil\right\rangle, T_{k}^{*} \cap T_{k+1}^{*}=\left\lceil e^{k}\right\rceil$, which is naturally called a rounded triad chain. In accordance with what has been said, the rounded triad chain consists of natural numbers and has the form:

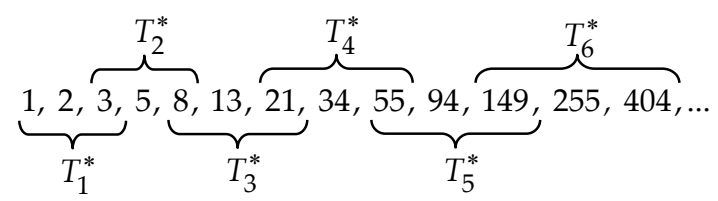

It is noteworthy that the first nine members of the sequence (A15), which are the union of four triads $\bigcup_{k=1}^{4} T_{k}^{*}$, are at the same time a fragment of the Fibonacci sequence $\left\{F_{n}\right\}, F_{n}=F_{n-2}+F_{n-1}$, $n \geq 2$, which has the form:

$$
0,1,1,2,3,5,8,13,21,34,55,89,144,233,377, \ldots
$$

That is,

$$
\left\{T_{k}^{*}\right\} \cap\left\{F_{n}\right\}=\{1,2,3,5,8,13,21,34,55\} .
$$

Analyzing this fact of intersection of the sequences (A15) and (A16), we note that the rounded triad chain (A15) is based on the syntropic section of the number $e$, while the limit of the ratio of the neighboring members of the Fibonacci sequence (A16) is equal to the number $\varphi$ [22], which is the quantitative characteristic of the golden ratio, that is, $\lim _{n \rightarrow \infty} \frac{F_{n+1}}{F_{n}}=\varphi, \varphi=\frac{1+\sqrt{5}}{2}=1.618 \ldots$. In other words, expression (A17) testifies to the close relations of the syntropic and golden ratios. We will show that this is indeed the case.

\section{A4. Syntropic and golden ratios}

The relationship between the quantity $\mathrm{a}$ and its parts $\mathrm{b}$ and $c=a-b$ in the case of the syntropic ratio is characterized by irrational numbers and, in the example of a left syntropic ratio, looks as follows. (Here and below, the values of irrational numbers are given with the accuracy of $\left.10^{-3}.\right)$

$$
\begin{gathered}
\frac{b}{a}=\frac{1}{e}=0.368, \\
\frac{c}{a}=\frac{e-1}{e}=0.632, \\
\frac{b}{c}=\frac{1}{e-1}=0.582 .
\end{gathered}
$$

Analysis of the above expressions shows that if the ratios (A19) and (A20) are rounded to $10^{-1}$, then we get an approximate expression of the golden ratio:

$$
\frac{b}{c} \approx \frac{c}{a} \approx 0.6
$$

In this regard, let us recall that the golden ratio is such a division of a quantity $a$ into two parts $b$ and $c=a-b$, for which the following proportion is satisfied:

$$
\frac{b}{c}=\frac{c}{a}=\frac{1}{\varphi}=0.618
$$

In addition, it follows from comparison (A19) and (A22) that the golden ratio lies within the interval of harmonious divisions (A13) at a distance of $0.014 a$ from the syntropic ratio. More clearly, this 
proximity of the ratios can also be represented if the division of an arbitrary quantity into two parts is expressed in rounded percentages. In this case, the golden ratio (A22) gives the value of the parts of $62 \%$ and $38 \%$, whereas the syntropic ratio, according to (A18) and (A19), $63 \%$ and $37 \%$. All this suggests that the syntropic and golden ratios are slightly different from each other and can be considered as alternative variants of the harmonious division of the whole into two parts.

\section{References}

1. Ursul, A.D. Reflection and information; Mysl: Moscow, Russia, 1973; 231 p.

2. Ukraintsev, B.S. Information and reflection. Questions of philosophy 1963, 2, 26-38. (In Russian)

3. Novik, I.B. Negentropy and the amount of information. Questions of philosophy 1962, 6, 118-128. (In Russian)

4. Pfaundler, M.; von Seht, L. Über Syntropie von Krankheitszuständen. Zeitschrift für Kinderheilkunde 1921, 30(1), 100-120.

5. Hartley, R.V.L. Transmission of Information. Bell System Technical Journal 1928, 7(3), 535-563.

6. Shannon, E.C. A mathematical theory of communication. Bell System Technical Journal 1948, 27, 379423.

7. Kolmogorov, A.N. Three approaches to the definition of the concept "quantity of information". Probl. Peredachi Inf. 1965, 1:1, 3-11.

8. Weaver, W. Recent contributions to the mathematical theory of communication. In The mathematical theory of communication; Shannon C.E., Weaver W., Eds.; The University of Illinois press: Urbana, USA, 1964, pp. 1-28.

9. Haken, H. Synergetics, an introduction. Nonequilibrium Phase-Transitions and Self-Organization in Physics, Chemistry and Biology, $2^{\text {nd }}$ ed.; Springer-Verlag Berlin Heidelberg: Berlin - New York, Germany USA, 1978.

10. Vyatkin, V.B. Mathematical models of informational assessment of the characteristics of ore objects. Dissertation for the Degree of Candidate of technical sciences, USTU-UPI, Ekaterinburg, Russia, 2004.

11. Vyatkin, V.B. Synergetic approach to defining the amount of information. Information Technologies 2009, 12, 68-73. (In Russian)

12. Vyatkin, V.B. Introduction to the synergetic theory of information. Information Technologies 2010, 12, 67-73. (In Russian)

13. Vyatkin, V.B. Integrative-code interrelation between combinatorial, probabilistic and synergetic approaches to defining the amount of information. Information Technologies 2016, 22(7), 542-548. (In Russian)

14. Vyatkin, V.B. Chaos and order of discrete systems in the light of the synergetic theory of information. Scientific journal of the Kuban State Agrarian University 2009, 47(3). (In Russian)

15. Tribus, M. Thermostatics and Thermodynamics; an Introduction to Energy, Information and States of Matter, with Engineering Applications; University Series in Basic Engineering, D. Van Nostrand Company, Inc., New York, USA, 1961.

16. Planck, M. Einführung in die theoretische Physik. Band V: Einführung in die Theorie der Wärme; Leipzig, 1930.

17. Kolmogorov, A.N. Combinatorial foundations of information theory and calculus of probabilities. Russian Mathematical Surveys 1983, 38(4), 27-36.

18. Kharkevich, A.A. Essays on the general theory of communication; Gostekhizdat: Moscow, Russia, 1955; $268 \mathrm{p}$.

19. Foerster, H. On Self-Organizing Systems and Their Environments. In Self-Organizing Systems, Yovits M.C., Cameron S. (Hg.), Eds.; Pergamon Press: London, 1960; pp. 31-50.

20. Petrik, E.A. Features of polymorbidity in somatic patients. Author's abstract of dissertation for the Degree of Candidate of medical sciences, MSUMD, Moscow, Russia, 2011.

21. Yaglom, A.M.; Yaglom I.M. Probability and Information. Nauka: Moscow, Russia, 1973; 512 p.

22. Dunlap, R.A. The Golden Ratio and Fibonacci Numbers. World Scientific Publishing Company: Singapore, $1998 ; 170 \mathrm{p}$. 